

\title{
AM1* parameters for gold
}

Hakan Kayi

\section{To cite this version:}

Hakan Kayi. AM1* parameters for gold. Journal of Molecular Modeling, 2009, 16 (5), pp.1029-1038. 10.1007/s00894-009-0613-z . hal-00568332

\section{HAL Id: hal-00568332 \\ https://hal.science/hal-00568332}

Submitted on 23 Feb 2011

HAL is a multi-disciplinary open access archive for the deposit and dissemination of scientific research documents, whether they are published or not. The documents may come from teaching and research institutions in France or abroad, or from public or private research centers.
L'archive ouverte pluridisciplinaire HAL, est destinée au dépôt et à la diffusion de documents scientifiques de niveau recherche, publiés ou non, émanant des établissements d'enseignement et de recherche français ou étrangers, des laboratoires publics ou privés. 


\section{Editorial Manager(tm) for Journal of Molecular Modeling Manuscript Draft}

Manuscript Number: JMM01049R1

Title: AM1* parameters for gold

Article Type: Original paper

Keywords: AM1*; Gold parameters; NDDO; Semiempirical MO-theory

Corresponding Author: Mr. Hakan Kayi, M.Sc.

Corresponding Author's Institution: Friedrich-Alexander Universität Erlangen-Nürnberg

First Author: Hakan Kayi, M.Sc.

Order of Authors: Hakan Kayi, M.Sc.

Abstract: We report the parameterization of AM1* for gold. The basis set for gold contains one set each of s-, p- and d orbitals. AM1* parameters are now available for $\mathrm{H}, \mathrm{C}, \mathrm{N}, \mathrm{O}$ and $\mathrm{F}$ (which use the original AM1 parameters), $\mathrm{Al}, \mathrm{Si}, \mathrm{P}, \mathrm{S}, \mathrm{Cl}, \mathrm{Ti}, \mathrm{V}, \mathrm{Cr}, \mathrm{Mn}, \mathrm{Fe}, \mathrm{Co}, \mathrm{Ni}, \mathrm{Cu}, \mathrm{Zn}, \mathrm{Br}, \mathrm{Zr}, \mathrm{Mo}$, I and $\mathrm{Au}$. The performance and typical errors of AM1* are discussed for gold.

Response to Reviewers: First of all, I would like to thank referees to much for very valuable comments.

Corrections done:

1) On the bottom of page 6 and the top of page 7, relative energies of isomers are modified as $\mathrm{kcal} / \mathrm{mol}$ (instead of eV).

2) In the first sentence of the Discussion, two subject-verb agreement errors with "have/has" and "provide/provides" are corrected.

Many thanks again for the comments. 

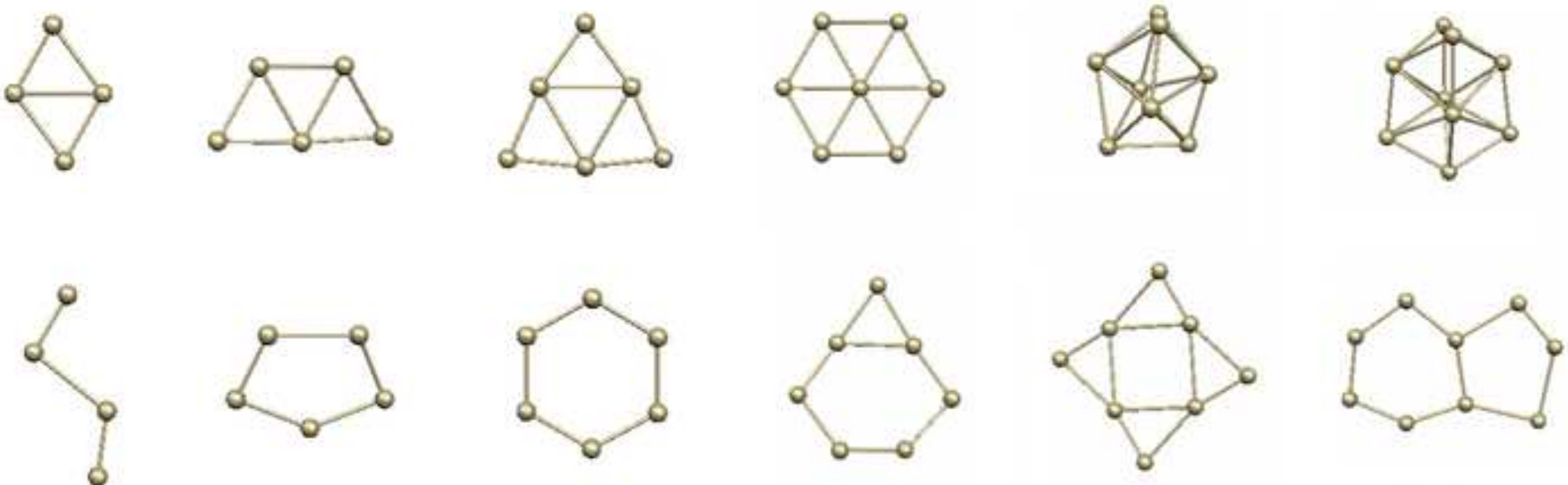


\title{
AM1* parameters for gold
}

Received: 08.10.2009 / Accepted: 22.10.2009

Hakan Kayi

Computer-Chemie-Centrum and Interdisciplinary Center for Molecular Materials, Friedrich-Alexander-Universität Erlangen-Nürnberg, Nägelsbachstraße 25, 91052 Erlangen, Germany

${ }^{\square}$ E-mail: Hakan.Kayi@ chemie.uni-erlangen.de

Dedicated to Professor Tim Clark on the occasion of his $60^{\text {th }}$ birthday

\begin{abstract}
We report the parameterization of AM1* for gold. The basis set for gold contains one set each of $s$-, $p$ - and $d$-orbitals. AM1* parameters are now available for $\mathrm{H}, \mathrm{C}, \mathrm{N}, \mathrm{O}$ and $\mathrm{F}$ (which use the original AM1 parameters), Al, Si, P, S, Cl, Ti, V, Cr, Mn, Fe, Co, Ni, Cu, Zn, Br, Zr, Mo, $\mathrm{I}$ and $\mathrm{Au}$. The performance and typical errors of AM1* are discussed for gold.
\end{abstract}

Keywords $\quad$ AM1* $\cdot$ Gold parameters $\cdot$ NDDO $\cdot$ Semiempirical MO-theory 


\section{Introduction}

AM1* [1-7] is an extension of AM1 [8] that uses $d$-orbitals for the elements $\mathrm{P}, \mathrm{S}, \mathrm{Cl}$ [1], $\mathrm{Al}$, $\mathrm{Si}$, Ti and $\mathrm{Zr}$ [2], $\mathrm{Cu}$ and $\mathrm{Zn}$ [3], Br and I [4], $\mathrm{V}$ and $\mathrm{Cr}$ [5], Co and Ni [6], Mn and Fe [7]. The AM1* molybdenum parameters are a slight modification of Voityuk and Rösch's AM1(d) parameter set [9]. AM1* retains the original AM1 parameters for the elements $\mathrm{H}, \mathrm{C}, \mathrm{N}, \mathrm{O}$ and F. The intention is to provide a technique that has the advantages of AM1 for first-row elements, such as good energies for hydrogen bonds, higher rotation barriers for $\pi$-systems than MNDO [10, 11] or PM3 [12-14] but performs better for heavier elements and to be applicable to transition metals. As a continuation of this work, new AM1* parameters for gold are now reported. Gold is important in the chemistry of catalysts and it is also very promising for biochemical and biomedical applications $[15,16]$. Because the experimental data for heats of formation of compounds of gold are relatively sparse, we have also used a series of model compounds whose heats of formation we have derived from DFT calculations [17].

\section{Theory}

AM1* for the new element gold uses the same basic theory as outlined previously [1,2]. As for other element- $\mathrm{H}$ interactions, the core-core repulsion potential for the $\mathrm{Au}-\mathrm{H}$ interaction used a distance-dependent term $\delta_{i j}$, rather than the constant term used for core-core potentials for most other interactions in AM1*. [1] This distance-dependent $\delta_{i j}$ was also used for the Mo-H and interaction in AM1(d) [8] and for Ti-H, V-H, Cr-H, Co-H, Ni-H, Cu-H, Zn-H, Br$\mathrm{H}, \mathrm{Zr}-\mathrm{H}, \mathrm{Mo}-\mathrm{H}$ and $\mathrm{I}-\mathrm{H}$ in AM1* [2-7]. The core-core term for $\mathrm{Au}-\mathrm{H}$ is thus:

$$
E^{c o r e}(i-j)=Z_{i} Z_{j} \rho_{s s}^{0}\left[1+r_{i j} \delta_{i j} \exp \left(-\alpha_{i j} r_{i j}\right)\right]
$$

where all terms have the same meaning as given in reference [1].

And, the standard MNDO/d formula is used for all other core-core interactions:

$$
E^{\text {core }}(i-j)=Z_{i} Z_{j} \rho_{s s}^{0}\left[1+\delta_{i j} \exp \left(-\alpha_{i j} r_{i j}\right)\right]
$$


The parameterization techniques were those reported in references [1] and [2] and will not be described further here.

\section{Parameterization data}

The target values used for parameterization and their sources are defined in Tables S1, S2 and S3 of the Supplementary Material. For the parameterization of the gold, we have used heats of formation, Koopmans' ionization potentials, dipole moments and geometrical variables as we did for the $\mathrm{Ti}$ and $\mathrm{Zr}$ [2], $\mathrm{Cu}$ and $\mathrm{Zn}$ [3], $\mathrm{Br}$ and I [4], $\mathrm{V}$ and $\mathrm{Cr}$ [5], $\mathrm{Co}$ and $\mathrm{Ni}$ [6], $\mathrm{Mn}$ and $\mathrm{Fe}$ [7] parameterizations. Parameterization data were extended with a small series of compounds whose target values derived from DFT calculations. As before [1-7], we also checked that experimental values for heats of formation were reasonable using DFT calculations.

DFT calculations used the Gaussian 03 suite of programs [18] with the LANL2DZ basis set and standard effective core potentials [19-22] augmented by a set of polarization functions [23] (designated LANL2DZ+pol) and the B3LYP hybrid functional [24-26].

Experimental parameterization data for gold were taken largely from the NIST Webbook [27], but also from the OpenMopac collection [28] and the other experimental and theoretical sources given in the Supplementary Material.

The energetic parameterization data and their sources are given in Table S1 of the Supplementary Material. In addition to the energetic data, geometries, dipole moments and ionization potentials taken from the above sources, crystal structures from the Cambridge Structural Database (CSD) [29] were used in the parameterization to ensure that not only the energetic and electronic properties for the "prototype" compounds, but also the structures of large gold compounds are well produced.

\section{Results}

The optimized AM1* parameters are shown in Table 1. Geometries were optimized with the new AM1* parameterization using VAMP 10.0 [30], while the PM6 calculations used MOPAC2007 [31]. The two programs give essentially identical results for the Hamiltonians that are available in both. 
- Table 1 about here -

\section{Heats of formation}

The calculated heats of formation for our training set of gold compounds are shown in Table 2. Results have been compared with the only comparable method available Stewart's PM6 [32].

- Table 2 about here -

AM1* reproduces the heats of formation of the training set, which consists of 49 gold compounds, used in parameterization better than PM6. AM1* shows no significant systematic error trend with a mean signed error (MSE) of $-0.7 \mathrm{kcal} \mathrm{mol}^{-1}$, whereas PM6 tends to overestimate heats of formation to gold compounds by $7.5 \mathrm{kcal} \mathrm{mol}^{-1}$. The mean unsigned error (MUE) between experimental and AM1*-calculated heats of formation is only $11.4 \mathrm{kcal}$ $\mathrm{mol}^{-1}$ and the root mean square deviation (RMSD) is $19.4 \mathrm{kcal} \mathrm{mol}^{-1}$. These values are smaller than those given by PM6 (MUE $=23.1 \mathrm{kcal} \mathrm{mol}^{-1}$ and RMSD $=33.7 \mathrm{kcal} \mathrm{mol}^{-1}$ ). PM6 produces large errors for the compounds that were not included in its original training set. For example, PM6 gives errors in heats of formation of more than $50 \mathrm{kcal} \mathrm{mol}^{-1}$ for the compounds $\mathrm{AuAlH}_{2}, \mathrm{AuPH}_{3}{ }^{+}, \mathrm{AuV}, \mathrm{AuNi}$ and $\mathrm{AuCu}$. However, Table 2 also shows the performance of AM1* and PM6 for only the PM6 parameterization dataset, which consists of 32 gold-containing compounds [32]. The AM1* mean unsigned error of only $10.7 \mathrm{kcal} \mathrm{mol}^{-1}$ and RMSD of $15.6 \mathrm{kcal} \mathrm{mol}^{-1}$ compared with $12.8 \mathrm{kcal} \mathrm{mol}^{-1}$ (MUE) and $15.9 \mathrm{kcal} \mathrm{mol}^{-1}$ (RMSD) for PM6 are very respectable and suggest that AM1* is parameterized well and also performs better than PM6 for the PM6 parameterization subset.

The large positive errors for AM1* are found for $\mathrm{Au}\left(\mathrm{CH}_{3}\right) \mathrm{F}_{2}\left(47.0 \mathrm{kcal} \mathrm{mol}{ }^{-1}\right), \mathrm{AuCl}_{2}{ }^{-}(31.6$ $\left.\mathrm{kcal} \mathrm{mol}^{-1}\right)$ and $\mathrm{Au}(\mathrm{CO})_{4}{ }^{+}\left(27.4 \mathrm{kcal} \mathrm{mol}^{-1}\right)$. The largest negative errors are found for AuZrH $\left(-81.9 \mathrm{kcal} \mathrm{mol}^{-1}\right), \mathrm{AuPH}_{3}{ }^{+}\left(-48.2 \mathrm{kcal} \mathrm{mol}^{-1}\right)$ and AuMo (-35.4 kcal mol$\left.{ }^{-1}\right)$. The large errors with $\mathrm{Au}\left(\mathrm{CH}_{3}\right) \mathrm{F}_{2}$ and $\mathrm{Au}(\mathrm{CO})_{4}{ }^{+}$are not very surprising, since $\mathrm{AM} 1 *$ uses the unchanged $\mathrm{AM} 1$ parameterization for the elements $\mathrm{H}, \mathrm{C}, \mathrm{N}, \mathrm{O}$ and $\mathrm{F}$, and this limits the possible accuracy of the parameterization. However, this does not explain the large error with $\mathrm{AuCl}_{2}^{-}, \mathrm{AuZrH}$, $\mathrm{AuPH}_{3}{ }^{+}$and AuMo. With the exception of the hydrogen in $\mathrm{AuZrH}$ and $\mathrm{AuPH}_{3}{ }^{+}$, these compounds contain only pure $\mathrm{AM} 1 *$ elements. As found for other metals, the large errors in these pure $\mathrm{AM} 1 *$ element-containing compounds is likely to be a consequence of our 
sequential parameterization strategy, in contrast to the simultaneous parameterization used for PM6 [32].

\section{Ionization potentials and dipole moments}

Table 3 shows a comparison of the calculated and experimental Koopmans' theorem ionization potentials and dipole moments for AM1* and PM6.

\section{- Table 3 about here -}

AM1* performs marginally better than PM6 for the ionization potentials with an MUE of 0.38 $\mathrm{eV}$ comparing with $1.19 \mathrm{eV}$ for PM6. Here, one must notice that all the experimental ionization potential data taken from NIST Webbook [27] and that no details of the performance for ionization potentials of gold compounds was given in the original PM6 parameterization of gold [32]. On the other hand, only $\mathrm{Au}$ and $\mathrm{Au}_{2}$ were used in the training set of $\mathrm{AM} 1^{*}$ parameterization and the remaining compounds were used for the test step. The largest $\mathrm{AM} 1 *$ errors, more than $0.5 \mathrm{eV}$, are found for $\mathrm{AuAl}, \mathrm{AuAl}_{2}$ and $\mathrm{Au}_{2} \mathrm{Al}(0.63,0.51$ and $0.70 \mathrm{eV}$, respectively). Both AM1* and PM6 tend to give positive systematic errors for ionization potentials of gold-containing compounds with MSEs of 0.13 and $0.25 \mathrm{eV}$, respectively.

The AM1* training set for dipole moments consists of 14 compounds taken directly from the PM6 parameterization dataset and was not extended. AM1* underestimates the dipole moments of gold-containing compounds by -0.33 Debye for this dataset, whereas PM6 tends to give positive systematic errors with an MSE of 0.21 Debye. The performance of the two methods is comparable for dipole moments. The mean unsigned errors are found to be 0.77 Debye and 0.78 Debye for AM1* and PM6, respectively. AM1* errors larger than 1.0 Debye, are found for the compounds $\mathrm{Au}\left(\mathrm{NH}_{2}\right)_{3}$ (-2.06 Debye), $\mathrm{AuI}$ (1.53 Debye), $\mathrm{Au}\left(\mathrm{CH}_{3}\right) \mathrm{F}_{2}(-1.50$ Debye), AuF (1.19 Debye), $\mathrm{Au}\left(\mathrm{CH}_{3}\right)_{3}$ (-1.13 Debye), $\mathrm{Au}\left(\mathrm{CH}_{3}\right)_{2}(\mathrm{OH})$ (-1.10 Debye). With the sole exception of AuI, these compounds contain the original AM1 elements $\mathrm{H}, \mathrm{C}, \mathrm{N}, \mathrm{O}$ and F, which limits the possible accuracy of the parameterization. The large error with AuI is also not very surprising as it is a result of the known weakness of AM1* parameterization for iodine in reproducing dipole moments [4]. 


\section{Geometries}

The geometrical parameters used to parameterize AM1* for gold and a comparison and statistical analysis of the AM1* and PM6 results are shown in Table 4.

\section{- Table 4 about here -}

AM1* shows no significant systematic error for gold with an MSE of $0.01 \AA$. In contrast to AM1*, PM6 with an MSE of $0.10 \AA$, predicts the bond lengths of the gold-containing compounds to be too long. AM1* with an MUE of $0.07 \AA$ performs far better than PM6 (MUE=0.28 $\AA$ ) for the training set, which consists of 75 compounds. Once again, as in heats of formations, large errors for PM6 originated from the compounds not included in the original PM6 parameterization dataset. For the bond angles, AM1* performs quite well $\left(\mathrm{MUE}=6.9^{\circ}\right)$ compared to PM6 $\left(\mathrm{MUE}=10.4^{\circ}\right)$. AM1* overestimates bond angles at gold systematically with an MSE of $3.7^{\circ}$, whereas PM6 underestimates them by $-2.1^{\circ}$. However, AM1* not only performs well for its own extended dataset, it also performs better for the PM6 parameterization subset which consists of 50 compounds [32]. For the PM6 training set, AM1* gives MUEs of $0.08 \AA$ for bond lengths and $7.5^{\circ}$ for bond angles, whereas PM6 gives MUEs of $0.15 \AA$ and $12.4^{\circ}$ for bond lengths and angles, respectively.

\section{- Fig.1 about here -}

The AM1* and PM6 optimized ground state structures of neutral gold clusters with 4 to 9 atoms are shown in Fig. 1. PM6 predicts neutral gold clusters up to 9 atoms to be planar as reported earlier by Stewart. [32] AM1* gives planar structures up to 7 atoms. AM1* finds non-planar 3D structures for gold clusters with more than 8 atoms, in good agreement with Häkkinen and Landman's results obtained from density-functional theory with scalarrelativistic ab initio pseudopotentials and a generalized gradient correction. [33] On the other hand, Gruene et al. [34] proposed a planar edge-capped triangle with $\mathrm{C}_{\mathrm{s}}$ symmetry to be lowest in energy for neutral $\mathrm{Au}_{7}$ on the strength of experimental vibrational spectra and DFT calculations within the generalized gradient approximation, in contrast to Häkkinen and Landman's hexagonal planar structure [33] and Wang et al.'s 3D pentagonal bipyramidal structure from DFT with local density approximation [35].

- Fig.2 about here - 
As shown in Fig. 2, AM1* reproduces the planar edge-capped triangle $\mathrm{Au}_{7}$ structure very well, but finds it to be $7.38 \mathrm{kcal} \mathrm{mol}^{-1}$ higher in energy than the hexagonal structure. PM6 does not reproduce the structure of this isomer structure very well, but finds it to be $0.23 \mathrm{kcal}$ $\mathrm{mol}^{-1}$ lower in energy than the hexagonal structure.

Very recently Botana et al. [36] performed an LCGTO-KSDFM density-functional theory [37] study and found that the global energy minimum for $\mathrm{Au}_{8}$ is a planar $\mathrm{D}_{4 \mathrm{~h}}$ tetra-capped square structure. A non-planar $\mathrm{D}_{2 \mathrm{~d}}$ bi-capped octahedron structure was found to be the second lowest energy structure. Wang et al. [35] proposed a $\mathrm{T}_{\mathrm{d}}$ bicapped octahedron structure using relativistic effective core potentials (ECP) and a double numerical basis with $d$-polarization functions. Their results are consistent with Häkkinen and Landman's generalized gradient approximation (GGA) results [33]. On the other hand, Olson and Gordon [38] found that a non-planar $\mathrm{D}_{2 \mathrm{~d}}$ bi-capped octahedron structure is the lowest energy neutral $\mathrm{Au}_{8}$ isomer, using Møller-Plesset perturbation theory (MP2) [39] regardless of basis set or contributions from core-valence correlation. They also found that CCSD [40] calculations (with some exceptions due to core-valence correlation effects) give tetra-capped square structure as the minimum energy structure.

Since there are many different proposals originating from different level of calculations, both AM1* and PM6 perform reasonably as well as far as we can tell.

\section{Discussion}

The new AM1* parameterization for gold, for which the parameterization dataset has been extended by including results from DFT calculations to produce a parameter set that is more robust and more reliable, provides an important additional element especially for catalytic chemistry and biochemistry applications. AM1* parameterization for gold gives very good results for energetic, electronic and structural properties. AM1* performs very well for its own training set and also for PM6 parameterization subset. In addition, for small neutral gold clusters, AM1* performs very well especially for the structural properties. Naturally, as for all semiempirical methods, there can be some cases that both AM1* and PM6 might show large deviations from experiment. As published NDDO-based methods including $d$-orbitals, both AM1* and PM6 provide opportunity to perform comparative calculations for different applications and good starting points for reaction specific local parameterizations. 


\section{Acknowledgments}

This paper is dedicated to Professor Tim Clark on the occasion of this $60^{\text {th }}$ birthday. I would like to thank him for his endless support, patience and for always encouraging me. I also thank Deutsche Forschungsgemeinschaft for financial support (by an individual grant (C185/17-1) and as part of GK312 "Homogeneous and Heterogeneous Electron Transfer" and SFB583 “Redox-Active Metal Complexes: Control of Reactivity via Molecular Architecture”).

\section{Supplementary material}

The values and the sources of the parameterization data. 


\section{References}

1. Winget P, Horn AHC, Selçuki C, Martin B, Clark T (2003) J Mol Model 9:408-414

2. Winget P, Clark T (2005) J Mol Model 11:439-456

3. Kayi H, Clark T (2007) J Mol Model 13:965-979

4. Kayi H, Clark T (2009) J Mol Model 15:295-308

5. Kayi H, Clark T (2009) J Mol Model 15:1253-1269

6. Kayi H, Clark T (2009) J Mol Model 15 (online first, DOI: 10.1007/s00894-009-0503-4)

7. Kayi H, Clark T (2009) J Mol Model 15 (submitted)

8. Dewar MJS, Zoebisch EG, Healy EF, Stewart JJP (1985) J Am Chem Soc 107:3902-3909

9. Voityuk AA, Rösch N (2000) J Phys Chem A 104:4089-4094

10. Dewar MJS, Thiel W (1977) J Am Chem Soc 99(15):4899-4907

11. Thiel W (1998) In: Schleyer PvR, Allinger NL, Clark T, Gasteiger J, Kollman PA, Schaefer III HF, Schreiner PR (eds) Encyclopedia of Computational Chemistry. Wiley, Chichester, p 1599

12. Stewart JJP (1989) J Comp Chem 10:209-220

13. Stewart JJP (1989) J Comp Chem 10:221-264

14. Stewart JJP (1998), In: Schleyer PvR, Allinger NL, Clark T, Gasteiger J, Kollman PA, Schaefer III HF, Schreiner PR (eds) Encyclopedia of Computational Chemistry. Wiley, Chichester, p 2080

15. Daniel MC, Astruc D (2004) Chem Rev 104:293-346

16. Shubina TE, Hartnig C, Koper MTM (2004) Phys Chem Chem Phys 6:4215-4221

17. Winget P, Clark T (2004) J Comp Chem 25:725-733

18. Frisch MJ, Trucks GW, Schlegel HB, Scuseria GE, Robb MA, Cheeseman JR, Montgomery JA Jr, Vreven T, Kudin KN, Burant JC, Millam JM, Iyengar SS, Tomasi J, Barone V, Mennucci B, Cossi M, Scalmani G, Rega N, Petersson GA, Nakatsuji H, Hada M, Ehara M, Toyota K, Fukuda R, Hasegawa J, Ishida M, Nakajima T, Honda Y, Kitao O, Nakai H, Klene M, Li X, Knox JE, Hratchian HP, Cross JB, Bakken V, Adamo C, Jaramillo J, Gomperts R, Stratmann RE, Yazyev O, Austin AJ, Cammi R, Pomelli C, Ochterski JW, Ayala PY, Morokuma K, Voth GA, Salvador P, Dannenberg JJ, Zakrzewski VG, Dapprich S, Daniels AD, Strain MC, Farkas O, Malick DK, Rabuck AD, Raghavachari K, Foresman JB, Ortiz JV, Cui Q, Baboul AG, Clifford S, Cioslowski J, Stefanov BB, Liu G, Liashenko A, Piskorz P, Komaromi I, Martin RL, Fox DJ, Keith T, 
Al-Laham MA, Peng CY, Nanayakkara A, Challacombe M, Gill PMW, Johnson B, Chen W, Wong MW, Gonzalez C, Pople JA (2004) Gaussian 03. Gaussian Inc, Wallingford CT 19. Dunning Jr TH, Hay PJ, (1976) In: Schaefer III HF (ed) Modern Theoretical Chemistry, Vol 3. Plenum, New York, pp 1-28

20. Hay PJ, Wadt WR (1985) J Chem Phys 82(1):270-283

21. Hay PJ, Wadt WR (1985) J Chem Phys 82(1):284-298

22. Hay PJ, Wadt WR (1985) J Chem Phys 82(1):299-310

23. Frisch MJ, Pople JA, Binkley JS (1984) J Chem Phys 80:3265-3269

24. Becke AD (1988) Phys Rev A 38:3098

25. Lee C, Yang W, Parr RG (1988) Phys Rev B 37:785

26. Becke AD (1993) J Chem Phys 98:5648-5652

27. NIST Chemistry WebBook, NIST Standard Reference Database Number 69, (http://webbook.nist.gov/), Linstrom P, Mallard W, National Institute of Standards and Technology: Gaithersburg MD, 20899, 2003

28. Stewart JJP, http://openmopac.net/files.html

29. Cambridge Structural Database, Version 5.28 (2007) Cambridge Crystallographic Data Centre, Cambridge, UK

30. Clark T, Alex A, Beck B, Chandrasekhar J, Gedeck P, Horn AHC, Hutter M, Martin B, Rauhut G, Sauer W, Schindler T, Steinke T (2008) Computer-Chemie-Centrum, Universität Erlangen-Nürnberg: Erlangen

31. Stewart JJP, (2007) MOPAC2007, Stewart Computational Chemistry, Colorado Springs, CO, USA; http://OpenMOPAC.net

32. Stewart JJP (2007) J Mol Model 13:1173-1213

33. Häkkinen H, Landman U (2000) Phys Rev B62:R2287-R2290

34. Gruene P, Rayner DM, Redlich B, Meer AFG, Lyon JT, Meijer G, Fielicke A (2008) Science 321:674-676

35. Wang J, Wang G, Zhao J (2002) Phys Rev B66:0354181-0354186

36. Botana J, Pereiro M, Baldomir D, Arias JE (2009) Theor Chem Account 122:297-304

37. St-Amant A, Salahub DR (1990) Chem Phys Lett 169:387-392

38. Olson RM, Gordon MS (2007) J Chem Phys 126:2143101-2143106

39. Møller C, Plesset MS (1934) Phys Rev 46:618-622

40. Purvis GD, Bartlett RJ (1982) J Chem Phys 76:1910-1918 
Table 1 AM1* parameters for $\mathrm{Au}$

\begin{tabular}{|c|c|}
\hline Parameter & $\mathrm{Au}$ \\
\hline $\mathrm{U}_{\mathrm{ss}}[\mathrm{eV}]$ & -87.9070798 \\
\hline $\mathrm{U}_{\mathrm{pp}}[\mathrm{eV}]$ & -105.2325082 \\
\hline $\mathrm{U}_{\mathrm{dd}}[\mathrm{eV}]$ & -86.3002150 \\
\hline$\zeta_{s}\left[\right.$ bohr $\left.^{-1}\right]$ & 2.9612383 \\
\hline$\zeta_{p}\left[b_{0 h r}^{-1}\right]$ & 2.0921916 \\
\hline$\zeta_{d}\left[\mathrm{bohr}^{-1}\right]$ & 4.2954046 \\
\hline$\beta_{\mathrm{s}}[\mathrm{eV}]$ & -10.5044093 \\
\hline$\beta_{p}[e V]$ & -2.9212915 \\
\hline$\beta_{\mathrm{d}}[\mathrm{eV}]$ & -26.0306918 \\
\hline $\mathrm{g}_{\mathrm{ss}}[\mathrm{eV}]$ & 12.8779541 \\
\hline$g_{p p}[e V]$ & 41.6610633 \\
\hline$g_{\mathrm{sp}}[\mathrm{eV}]$ & 10.4094935 \\
\hline$g_{\mathrm{p} 2}[\mathrm{eV}]$ & 37.2533670 \\
\hline $\mathrm{h}_{\mathrm{sp}}[\mathrm{eV}]$ & 0.2611095 \\
\hline$z_{s n}\left[b o h r^{-1}\right]$ & 3.3536714 \\
\hline $\mathrm{z}_{\mathrm{pn}}\left[\mathrm{bohr}^{-1}\right]$ & 7.8813347 \\
\hline $\mathrm{z}_{\mathrm{dn}}\left[\mathrm{bohr}^{-1}\right]$ & 1.7991401 \\
\hline$\rho($ core $)\left[\right.$ bohr $\left.{ }^{-1}\right]$ & 1.3923445 \\
\hline$\Delta \mathrm{H}_{\mathrm{f}}^{\circ}($ atom $)\left[\mathrm{kcal} \mathrm{mol}{ }^{-1}\right]$ & 88.0 \\
\hline $\mathrm{F}_{s d}^{0}[\mathrm{eV}]$ & 7.7583836 \\
\hline $\mathrm{G}_{\mathrm{sd}}^{2}[\mathrm{eV}]$ & 6.0090661 \\
\hline \multicolumn{2}{|l|}{$\alpha(i j)$} \\
\hline H & 2.6891239 \\
\hline C & 3.1012172 \\
\hline $\mathbf{N}$ & 2.5099816 \\
\hline 0 & 3.3201280 \\
\hline $\mathbf{F}$ & 2.5990722 \\
\hline Al & 1.9195605 \\
\hline Si & 1.4489186 \\
\hline $\mathbf{P}$ & 3.3822499 \\
\hline S & 2.4757345 \\
\hline Cl & 2.5963772 \\
\hline $\mathrm{Ti}$ & 0.8968304 \\
\hline V & 3.9464525 \\
\hline $\mathrm{Cr}$ & 2.0645066 \\
\hline Mn & 3.0829513 \\
\hline $\mathrm{Fe}$ & 2.8842586 \\
\hline Co & 2.4940667 \\
\hline $\mathbf{N i}$ & 3.9231603 \\
\hline $\mathrm{Cu}$ & 2.2444292 \\
\hline Zn & 4.3646875 \\
\hline $\mathrm{Br}$ & 2.2685919 \\
\hline $\mathrm{Zr}$ & 1.3454899 \\
\hline Mo & 1.3740902 \\
\hline I & 4.3466246 \\
\hline $\mathrm{Au}$ & 2.7869271 \\
\hline \multicolumn{2}{|l|}{$\delta(\mathbf{i j})$} \\
\hline H & -2.3071373 \\
\hline C & 7.7686410 \\
\hline $\mathbf{N}$ & 2.0238749 \\
\hline
\end{tabular}




\begin{tabular}{|c|c|}
\hline $\mathbf{O}$ & 8.1526001 \\
$\mathbf{F}$ & 2.0413648 \\
$\mathbf{A l}$ & 1.6916486 \\
$\mathbf{S i}$ & 0.2684171 \\
$\mathbf{P}$ & 22.0561933 \\
$\mathbf{S}$ & 2.0695945 \\
$\mathbf{C l}$ & 3.0310645 \\
$\mathbf{T i}$ & 0.2436297 \\
$\mathbf{V}$ & 195.3767955 \\
$\mathbf{C r}$ & 1.7484764 \\
$\mathbf{M n}$ & 10.1393842 \\
$\mathbf{F e}$ & 7.0870550 \\
$\mathbf{C o}$ & 3.7260808 \\
$\mathbf{N i}$ & 51.9934681 \\
$\mathbf{C u}$ & 1.4743149 \\
$\mathbf{Z n}$ & 51.6768894 \\
$\mathbf{B r}$ & 1.2652966 \\
$\mathbf{Z r}$ & 0.5332656 \\
$\mathbf{M o}$ & 0.6104390 \\
$\mathbf{I}$ & 178.8807263 \\
$\mathbf{A u}$ & 6.0750313 \\
\hline
\end{tabular}


13

1 Table 2 Calculated AM1* and PM6 heats of formation and errors compared with our target values for the gold-containing compounds used to parameterize

2 AM1* (all values $\mathrm{kcal} \mathrm{mol}^{-1}$ ). Errors are classified by coloring the boxes in which they appear. Green indicates errors lower than $10 \mathrm{kcal}$ mol ${ }^{-1}$, yellow $10-20$

$3 \mathrm{kcal} \mathrm{mol}^{-1}$ and pink those greater than $20 \mathrm{kcal} \mathrm{mol}^{-1}$. The codenames within parentheses indicate the CSD-names of the compounds

\begin{tabular}{|c|c|c|c|c|c|}
\hline \multirow[b]{2}{*}{ Compound } & \multirow{2}{*}{$\begin{array}{c}\text { Target } \\
\Delta \mathbf{H}^{\circ}{ }_{f}^{\circ}\end{array}$} & \multicolumn{2}{|c|}{ AM1* } & \multicolumn{2}{|c|}{ PM6 } \\
\hline & & $\Delta \mathbf{H}_{\mathrm{f}}^{\circ}$ & Error & $\Delta \mathbf{H}^{\circ}{ }_{f}$ & Error \\
\hline $\mathrm{Au}$ & 88.0 & 88.0 & 0.0 & 77.5 & -10.5 \\
\hline $\mathrm{Au}^{-}$ & 20.0 & 8.3 & -11.7 & 33.0 & 13.0 \\
\hline $\mathrm{Au}^{+}$ & 300.7 & 286.0 & -14.7 & 286.6 & -14.1 \\
\hline $\mathrm{Au}_{2}$ & 87.0 & 114.1 & 27.1 & 84.7 & -2.3 \\
\hline AuH & 78.7 & 82.5 & 3.8 & 67.5 & -11.2 \\
\hline $\mathrm{AuH}_{3}$ & 149.1 & 128.7 & -20.5 & 136.5 & -12.6 \\
\hline $\mathrm{AuCH}_{3}$ & 67.3 & 73.1 & 5.8 & 77.4 & 10.1 \\
\hline $\mathrm{Au}\left(\mathrm{CH}_{3}\right)_{3}$ & 84.7 & 88.1 & 3.4 & 61.8 & -22.9 \\
\hline $\mathrm{AuNH}_{2}$ & 93.9 & 96.1 & 2.2 & 95.8 & 1.9 \\
\hline $\mathrm{Au}\left(\mathrm{CH}_{3}\right)_{2}\left(\mathrm{NH}_{2}\right)$ & 83.9 & 98.8 & 14.9 & 99.7 & 15.8 \\
\hline $\mathrm{Au}(\mathrm{CN})_{2}^{-}$ & 54.9 & 50.5 & -4.5 & 65.5 & 10.6 \\
\hline $\mathrm{Au}\left(\mathrm{NH}_{2}\right)_{3}$ & 126.0 & 121.9 & -4.1 & 131.3 & 5.3 \\
\hline $\mathrm{Au}\left(\mathrm{NH}_{3}\right)_{4}^{+}$ & 154.5 & 174.1 & 19.6 & 166.6 & 12.1 \\
\hline $\mathrm{Au}\left(\mathrm{NH}_{3}\right)_{6}{ }^{2+}$ & 355.1 & 346.9 & -8.2 & 367.0 & 11.9 \\
\hline$\left(\mathrm{Au}\left(\mathrm{CH}_{3}\right)_{2}(\mathrm{CN})\right)_{4}$ & 362.7 & 361.3 & -1.4 & 364.2 & 1.5 \\
\hline $\mathrm{AuO}^{-}$ & 57.1 & 54.4 & -2.7 & 46.0 & -11.1 \\
\hline $\mathrm{AuOH}$ & 57.8 & 46.0 & -11.8 & 50.2 & -7.6 \\
\hline $\mathrm{Au}\left(\mathrm{CH}_{3}\right)_{2}(\mathrm{OH})$ & 45.7 & 45.2 & -0.5 & 66.9 & 21.2 \\
\hline $\mathrm{Au}\left(\mathrm{H}_{2} \mathrm{O}\right)_{4}^{+}$ & -37.8 & -43.7 & -5.9 & 9.7 & 47.5 \\
\hline $\mathrm{Au}(\mathrm{CO})_{4}^{+}$ & 141.1 & 168.5 & 27.4 & 108.8 & -32.3 \\
\hline AuF & 50.0 & 55.4 & 5.4 & 83.6 & 33.6 \\
\hline $\mathrm{AuF}_{3}$ & 12.7 & 12.7 & 0.0 & 39.1 & 26.4 \\
\hline $\mathrm{AuF}_{4}^{-}$ & -79.7 & -82.5 & -2.8 & -113.5 & -33.8 \\
\hline $\mathrm{Au}\left(\mathrm{CH}_{3}\right) \mathrm{F}_{2}$ & -10.0 & 37.0 & 47.0 & 25.4 & 35.4 \\
\hline $\mathrm{AuAlH}_{2}$ & 90.6 & 90.5 & -0.1 & 163.8 & 73.2 \\
\hline $\mathrm{AuSiH}_{3}$ & 84.1 & 84.1 & 0.0 & 125.1 & 41.0 \\
\hline $\mathrm{AuPH}_{3}{ }^{+}$ & 260.8 & 212.6 & -48.2 & 192.6 & -68.2 \\
\hline $\mathrm{AuSH}_{2}^{+}$ & 269.1 & 246.6 & -22.5 & 243.2 & -25.9 \\
\hline $\mathrm{AuF}_{2}(\mathrm{SH})$ & 7.6 & 17.0 & 9.4 & 23.2 & 15.6 \\
\hline $\mathrm{Au}\left(\mathrm{Me}_{2} \mathrm{~S}\right)_{2}$ & 148.8 & 148.6 & -0.2 & 154.6 & 5.8 \\
\hline
\end{tabular}




$\mathrm{AuCl}^{-}$
$\mathrm{AuCl}_{2}^{-}$
$\mathrm{AuCl}_{3}$
$\mathrm{AuCl}_{4}^{-}$
$\mathrm{AuTi}^{-}$
$\mathrm{AuV}$
$\mathrm{AuCr}$
$\mathrm{AuCo}$
$\mathrm{AuNi}$
$\mathrm{AuCu}$
$\mathrm{AuZnH}$
$\mathrm{AuBr}$
$\mathrm{AuBr}$
2
$\mathrm{AuBr}_{4}^{-}$
$\mathrm{AuZrH}^{-}$
$\mathrm{AuMo}^{-}$
$\mathrm{Aul}^{-}$
$\mathrm{Aul}_{2}^{-}$
$\mathrm{Aul}_{4}^{-}$

$N=49$

Most positive error

Most negative error

MSE

MUE

RMSD

14

\begin{tabular}{rr|r|r|r|}
60.2 & 67.6 & 7.4 & 69.5 & 9.3 \\
-67.1 & -35.5 & 31.6 & -70.4 & -3.3 \\
65.1 & 51.4 & -13.7 & 58.0 & -7.1 \\
-89.7 & -89.7 & 0.0 & -92.9 & -3.2 \\
\hline 145.7 & 145.7 & 0.0 & 111.2 & -34.5 \\
100.2 & 107.0 & 6.8 & 203.3 & 103.1 \\
137.0 & 136.1 & -0.9 & 173.2 & 36.2 \\
\hline 141.5 & 141.6 & 0.1 & 132.4 & -9.1 \\
\hline 77.8 & 77.8 & 0.0 & 178.8 & 101.0 \\
117.7 & 117.7 & 0.0 & 200.1 & 82.4 \\
\hline 94.5 & 94.5 & 0.0 & 82.7 & -11.8 \\
62.8 & 68.7 & 5.9 & 68.9 & 6.1 \\
-56.3 & -50.9 & 5.4 & -50.1 & 6.2 \\
-66.4 & -71.5 & -5.1 & -70.0 & -3.6 \\
\hline 173.0 & 91.1 & -81.9 & 166.7 & -6.3 \\
\hline 251.5 & 216.1 & -35.4 & 209.6 & -41.9 \\
\hline 69.0 & 92.5 & 23.5 & 79.0 & 10.0 \\
-38.1 & -22.5 & 15.6 & -24.5 & 13.6 \\
-26.8 & -27.9 & -1.1 & -37.5 & -10.7 \\
& AM1* & & PM6 &
\end{tabular}

\begin{tabular}{|c|c|}
\hline 47.0 & 103.1 \\
\hline-81.9 & -68.2 \\
\hline-0.7 & 7.5 \\
\hline 11.4 & 23.1 \\
\hline 19.4 & 33.7 \\
\hline
\end{tabular}

Results for the PM6 parameterization set $(N=32)$

MSE

MUE

RMSD

\begin{tabular}{|cc|}
\hline 5.2 & 2.4 \\
10.7 & 12.8 \\
15.6 & 15.9 \\
\hline
\end{tabular}


7 Table 3 Calculated AM1* and PM6 Koopmans' theorem ionization potentials and dipole moments for gold-containing compounds. The errors are 8 color coded as follows: green up to $0.5 \mathrm{eV}$ or 0.5 Debye; yellow between 0.5 and 1.0 ; pink larger than 1.0

\begin{tabular}{|c|c|c|c|c|c|}
\hline \multirow{2}{*}{ Compound } & \multicolumn{3}{|c|}{ AM1* } & \multicolumn{2}{|c|}{ PM6 } \\
\hline & Target & \multicolumn{2}{|c|}{ Error } & \multicolumn{2}{|c|}{ Error } \\
\hline \multicolumn{6}{|c|}{ Koopmans' Theorem lonization Potentials for Cobalt Compounds (eV) } \\
\hline $\mathrm{Au}$ & 9.22 & 9.42 & 0.20 & 9.07 & -0.15 \\
\hline $\mathrm{Au}_{2}$ & 8.70 & 8.69 & -0.01 & 11.42 & 2.72 \\
\hline AuAl & 7.60 & 8.23 & 0.63 & 8.01 & 0.41 \\
\hline $\mathrm{AuAl}_{2}$ & 6.20 & 6.71 & 0.51 & 5.79 & -0.41 \\
\hline $\mathrm{Au}_{2} \mathrm{Al}$ & 7.70 & 8.40 & 0.70 & 8.89 & 1.19 \\
\hline AuSi & 9.50 & 9.17 & -0.33 & 7.72 & -1.78 \\
\hline $\mathrm{Au}\left(\mathrm{CH}_{3}\right) \mathrm{P}\left(\mathrm{CH}_{3}\right)_{3}$ & 7.70 & 7.26 & -0.44 & 6.27 & -1.43 \\
\hline \multirow{2}{*}{$\mathrm{Au}\left(\mathrm{CH}_{3}\right)_{3} \mathrm{P}\left(\mathrm{CH}_{3}\right)_{3}$} & 7.80 & 7.55 & -0.25 & 9.23 & 1.43 \\
\hline & & \multicolumn{2}{|c|}{ AM1* } & \multicolumn{2}{|c|}{ PM6 } \\
\hline \multicolumn{6}{|l|}{$N=8$} \\
\hline MSE & & \multicolumn{2}{|c|}{0.13} & \multicolumn{2}{|c|}{0.25} \\
\hline MUE & & \multicolumn{2}{|c|}{0.38} & \multicolumn{2}{|c|}{1.19} \\
\hline \multicolumn{6}{|c|}{ Dipole Moments for Cobalt Compounds (Debye) } \\
\hline $\mathrm{AuH}$ & 0.95 & 1.16 & 0.21 & 0.08 & -0.87 \\
\hline $\mathrm{AuCH}_{3}$ & 0.46 & 0.05 & -0.41 & 0.38 & -0.08 \\
\hline $\mathrm{Au}\left(\mathrm{CH}_{3}\right)_{3}$ & 1.78 & 0.65 & -1.13 & 0.41 & -1.37 \\
\hline $\mathrm{AuNH}_{2}$ & 1.63 & 1.70 & 0.07 & 2.73 & 1.10 \\
\hline $\mathrm{Au}\left(\mathrm{CH}_{3}\right)_{2}\left(\mathrm{NH}_{2}\right)$ & 0.34 & 0.42 & 0.08 & 1.19 & 0.85 \\
\hline $\mathrm{Au}\left(\mathrm{NH}_{2}\right)_{3}$ & 3.02 & 0.96 & -2.06 & 5.76 & 2.74 \\
\hline $\mathrm{AuOH}$ & 2.53 & 3.31 & 0.78 & 2.62 & 0.09 \\
\hline $\mathrm{Au}\left(\mathrm{CH}_{3}\right)_{2}(\mathrm{OH})$ & 3.74 & 2.64 & -1.10 & 3.04 & -0.70 \\
\hline AuF & 4.04 & 2.85 & -1.19 & 5.47 & 1.43 \\
\hline $\mathrm{Au}\left(\mathrm{CH}_{3}\right) \mathrm{F}_{2}$ & 2.46 & 0.96 & -1.50 & 2.85 & 0.39 \\
\hline $\mathrm{AuF}_{2}(\mathrm{SH})$ & 1.60 & 1.32 & -0.28 & 1.93 & 0.33 \\
\hline $\mathrm{AuCl}$ & 3.48 & 3.86 & 0.38 & 3.35 & -0.13 \\
\hline $\mathrm{AuBr}$ & 2.87 & 2.89 & 0.02 & 2.11 & -0.76 \\
\hline \multirow[t]{2}{*}{ Aul } & 2.21 & 3.74 & 1.53 & 2.11 & -0.10 \\
\hline & & \multicolumn{2}{|c|}{ AM1* } & \multicolumn{2}{|c|}{ PM6 } \\
\hline \multicolumn{6}{|l|}{$N=14$} \\
\hline MSE & & \multicolumn{2}{|c|}{-0.33} & \multicolumn{2}{|c|}{0.21} \\
\hline MUE & & \multicolumn{2}{|c|}{0.77} & \multicolumn{2}{|c|}{0.78} \\
\hline
\end{tabular}


16

10 Table 4 Calculated AM1* and PM6 bond lengths and angles for gold- containing compounds. The codenames within parentheses indicate the CSD-

11 names of the compounds. The errors are color coded as follows: green up to $0.05 \AA$ or $0.5^{\circ}$; yellow between $0.05-0.1 \AA$ or $0.5-1.0^{\circ}$; pink larger than $0.1 \AA^{\circ}$ or $1^{\circ}$ 12

\begin{tabular}{|c|c|c|c|c|c|c|}
\hline \multirow{2}{*}{ Compound } & \multirow{3}{*}{$\begin{array}{l}\text { Variable } \\
\text { Au-Au }\end{array}$} & \multirow[b]{2}{*}{ Target } & \multicolumn{2}{|c|}{ AM1* } & \multicolumn{2}{|c|}{ PM6 } \\
\hline & & & \multicolumn{2}{|c|}{ Error } & \multicolumn{2}{|c|}{ Error } \\
\hline $\mathrm{Au}_{2}$ & & 2.63 & 2.63 & 0.00 & 2.24 & -0.39 \\
\hline $\mathrm{Au}_{2}^{-}$ & $\mathrm{Au}-\mathrm{Au}$ & 2.73 & 2.67 & -0.07 & 2.49 & -0.24 \\
\hline AuH & $\mathrm{Au}-\mathrm{H}$ & 1.52 & 1.52 & 0.00 & 1.53 & 0.01 \\
\hline \multirow[t]{2}{*}{$\mathrm{AuH}_{3}$} & $\mathrm{Au}-\mathrm{H}$ & 1.70 & 1.58 & -0.13 & 1.71 & 0.01 \\
\hline & $\mathrm{H}-\mathrm{Au}-\mathrm{H}$ & 120.0 & 119.4 & -0.6 & 120.0 & 0.0 \\
\hline \multirow[t]{2}{*}{$\mathrm{AuCH}_{3}$} & $\mathrm{Au}-\mathrm{C}$ & 2.08 & 2.11 & 0.03 & 2.07 & -0.01 \\
\hline & $\mathrm{H}-\mathrm{C}-\mathrm{Au}$ & 107.2 & 107.3 & 0.1 & 107.1 & -0.1 \\
\hline \multirow[t]{3}{*}{$\mathrm{Au}\left(\mathrm{CH}_{3}\right)_{3}$} & $\mathrm{Au}-\mathrm{C}$ & 2.06 & 2.10 & 0.04 & 2.00 & -0.06 \\
\hline & $\mathrm{Au}-\mathrm{C}^{\prime}$ & 2.12 & 2.11 & -0.01 & 2.02 & -0.11 \\
\hline & C-Au-C & 176.1 & 160.1 & -16.0 & 169.4 & -6.7 \\
\hline $\mathrm{AuNH}_{2}$ & Au-N & 2.09 & 2.00 & -0.09 & 1.53 & -0.56 \\
\hline \multirow{3}{*}{$\mathrm{Au}\left(\mathrm{CH}_{3}\right)_{2}\left(\mathrm{NH}_{2}\right)$} & $\mathrm{Au}-\mathrm{C}$ & 2.13 & 2.10 & -0.03 & 2.06 & -0.07 \\
\hline & C-Au-C & 80.1 & 86.5 & 6.4 & 105.3 & 25.2 \\
\hline & $\mathrm{Au}-\mathrm{N}$ & 2.04 & 1.99 & -0.05 & 1.60 & -0.44 \\
\hline \multirow{2}{*}{$\mathrm{Au}(\mathrm{CN})_{2}^{-}$} & Au-C & 2.04 & 2.08 & 0.04 & 2.09 & 0.05 \\
\hline & C-Au-C & 180.0 & 180.0 & 0.0 & 180.0 & 0.0 \\
\hline \multirow[t]{2}{*}{$\mathrm{Au}(\mathrm{CN})_{2}^{-}(\mathrm{JEYXAM})$} & Au-C & 2.02 & 2.05 & 0.03 & 2.09 & 0.07 \\
\hline & $\mathrm{C}-\mathrm{N}$ & 1.11 & 1.16 & 0.05 & 1.15 & 0.04 \\
\hline \multirow{2}{*}{$\mathrm{AuC}_{3} \mathrm{~N}_{2} \mathrm{H}_{3}$ (CYMIAU) } & $\mathrm{Au}-\mathrm{C}(\mathrm{N})$ & 2.00 & 2.13 & 0.13 & 2.05 & 0.05 \\
\hline & $\mathrm{Au}-\mathrm{C}(\mathrm{NCH} 3)$ & 1.98 & 2.05 & 0.07 & 2.15 & 0.17 \\
\hline \multirow[t]{3}{*}{$\mathrm{Au}\left(\mathrm{NH}_{2}\right)_{3}$} & Au-N & 2.08 & 1.99 & -0.09 & 1.42 & -0.66 \\
\hline & $\mathrm{N}-\mathrm{Au}-\mathrm{N}$ & 158.1 & 156.0 & -2.1 & 139.3 & -18.9 \\
\hline & $\mathrm{Au}-\mathrm{N}^{\prime}$ & 2.11 & 2.05 & -0.06 & 2.03 & -0.08 \\
\hline $\mathrm{Au}\left(\mathrm{NH}_{3}\right)_{4}{ }^{3+}$ & Au-N & 2.17 & 2.17 & 0.00 & 2.14 & -0.03 \\
\hline $\mathrm{Au}\left(\mathrm{NH}_{3}\right)_{4}{ }^{+}$ & Au-N & 2.50 & 2.56 & 0.06 & 2.35 & -0.15 \\
\hline \multirow[t]{3}{*}{$\mathrm{AuC}_{14} \mathrm{~N}_{4} \mathrm{H}_{22}{ }^{+}$(AZEGIV) } & $\mathrm{Au}-\mathrm{N}$ & 1.99 & 2.03 & 0.04 & 2.14 & 0.15 \\
\hline & $\mathrm{Au}-\mathrm{N}^{\prime}$ & 1.97 & 2.03 & 0.06 & 2.02 & 0.05 \\
\hline & N-Au-N & 95.6 & 94.7 & -0.9 & 86.2 & -9.4 \\
\hline \multirow{2}{*}{$\mathrm{Au}\left(\mathrm{NH}_{3}\right)_{6}{ }^{2+}$} & $\mathrm{Au}-\mathrm{N}$ & 2.67 & 2.47 & -0.20 & 2.47 & -0.20 \\
\hline & Au-N' & 2.24 & 2.45 & 0.21 & 2.61 & 0.37 \\
\hline \multirow[t]{2}{*}{$\mathrm{Au}_{2} \mathrm{C}_{6} \mathrm{~N}_{2} \mathrm{H}_{2} \mathrm{O}$ (DEVLUL) } & Au-N & 2.14 & 2.21 & 0.07 & 2.18 & 0.04 \\
\hline & Au-C & 2.04 & 2.10 & 0.06 & 2.00 & -0.04 \\
\hline
\end{tabular}




\begin{tabular}{|c|c|c|c|c|c|c|}
\hline & C-Au-N & 97.1 & 93.2 & -3.9 & 95.2 & -1.9 \\
\hline \multirow[t]{4}{*}{$\left(\mathrm{Au}\left(\mathrm{CH}_{3}\right)_{2}(\mathrm{CN})\right)_{4}$} & $\mathrm{Au}-\mathrm{Au}$ & 5.50 & 5.53 & 0.03 & 5.53 & 0.03 \\
\hline & $\mathrm{Au}-\mathrm{C}(\mathrm{CN})$ & 2.03 & 2.10 & 0.07 & 2.11 & 0.08 \\
\hline & $A u-N$ & 2.14 & 2.22 & 0.08 & 2.28 & 0.14 \\
\hline & $\mathrm{Au}-\mathrm{C}(\mathrm{Me})$ & 2.11 & 2.15 & 0.04 & 1.98 & -0.13 \\
\hline $\mathrm{AuO}^{-}$ & $\mathrm{Au}-\mathrm{O}$ & 1.98 & 1.98 & 0.00 & 1.43 & -0.55 \\
\hline $\mathrm{AuOH}$ & $\mathrm{Au}-\mathrm{O}$ & 1.98 & 2.08 & 0.10 & 1.42 & -0.56 \\
\hline \multirow[t]{3}{*}{$\mathrm{Au}\left(\mathrm{CH}_{3}\right)_{2}(\mathrm{OH})$} & $\mathrm{Au}-\mathrm{O}$ & 2.09 & 2.10 & 0.01 & 1.88 & -0.21 \\
\hline & $\mathrm{Au}-\mathrm{C}$ & 2.10 & 2.10 & 0.00 & 2.00 & -0.10 \\
\hline & O-Au-C & 106.0 & 135.1 & 29.1 & 98.2 & -7.8 \\
\hline $\mathrm{Au}\left(\mathrm{H}_{2} \mathrm{O}\right)_{2}^{+}$ & $\mathrm{O}-\mathrm{Au}-\mathrm{O}$ & 176.8 & 172.1 & -4.7 & 176.9 & 0.1 \\
\hline \multirow[t]{2}{*}{$\mathrm{Au}\left(\mathrm{H}_{2} \mathrm{O}\right)_{4}^{+}$} & $\mathrm{Au}-\mathrm{O}$ & 2.46 & 2.46 & 0.00 & 2.39 & -0.07 \\
\hline & Au-O' & 2.45 & 2.45 & 0.00 & 2.36 & -0.09 \\
\hline $\mathrm{Au}(\mathrm{CO})_{4}^{+}$ & $A u-C$ & 2.28 & 2.34 & 0.06 & 2.30 & 0.02 \\
\hline AuF & Au-F & 2.07 & 1.95 & -0.12 & 1.92 & -0.15 \\
\hline \multirow[t]{3}{*}{$\mathrm{Au}\left(\mathrm{CH}_{3}\right) \mathrm{F}_{2}$} & $A u-F$ & 1.96 & 1.98 & 0.02 & 2.06 & 0.11 \\
\hline & Au-C & 2.05 & 2.04 & -0.01 & 1.94 & -0.11 \\
\hline & C-Au-F & 91.6 & 99.7 & 8.1 & 96.7 & 5.1 \\
\hline \multirow[t]{3}{*}{$\mathrm{AuF}_{3}$} & Au-F & 2.01 & 2.04 & 0.03 & 2.04 & 0.03 \\
\hline & $\mathrm{Au}-\mathrm{F}^{\prime}$ & 2.02 & 1.98 & -0.04 & 1.94 & -0.08 \\
\hline & F-Au-F' & 95.0 & 99.2 & 4.2 & 102.5 & 7.5 \\
\hline $\mathrm{AuF}_{4}^{-}$ & Au-F & 1.97 & 2.00 & 0.03 & 2.03 & 0.07 \\
\hline $\mathrm{AuAlH}_{2}$ & Au-Al & 2.38 & 2.42 & 0.03 & 3.01 & 0.63 \\
\hline $\mathrm{AuSiH}_{3}$ & $\mathrm{Au}-\mathrm{Si}$ & 2.32 & 2.32 & 0.00 & 3.38 & 1.06 \\
\hline $\mathrm{AuPH}_{3}{ }^{+}$ & Au-P & 2.28 & 2.39 & 0.11 & 2.11 & -0.17 \\
\hline $\mathrm{AuPH}_{2}$ & Au-P & 2.33 & 2.28 & -0.05 & 2.09 & -0.24 \\
\hline $\mathrm{AuP}^{2-}$ & $A u \equiv P$ & 2.28 & 2.20 & -0.08 & 1.59 & -0.69 \\
\hline $\mathrm{Au}\left(\mathrm{PH}_{3}\right)_{2}^{+}$ & $A u-P$ & 2.38 & 2.34 & -0.04 & 2.20 & -0.18 \\
\hline \multirow{2}{*}{$\mathrm{Au}_{2} \mathrm{C}_{10} \mathrm{P}_{4} \mathrm{H}_{28}{ }^{+}$(DUKREG01) } & Au-P & 2.30 & 2.39 & 0.09 & 2.39 & 0.09 \\
\hline & P-Au-P & 176.8 & 177.0 & 0.3 & 91.0 & -85.8 \\
\hline AuSH & $\mathrm{Au}-\mathrm{S}$ & 2.30 & 2.29 & -0.01 & 2.18 & -0.12 \\
\hline $\mathrm{AuSH}_{2}^{+}$ & Au-S & 2.37 & 2.48 & 0.12 & 2.13 & -0.24 \\
\hline \multirow[t]{4}{*}{$\mathrm{Au}_{2} \mathrm{C}_{8} \mathrm{H}_{22} \mathrm{~S}_{2}(\mathrm{CULYIR})$} & $A u-A u$ & 3.46 & 3.59 & 0.13 & 3.51 & 0.05 \\
\hline & Au-S & 2.41 & 2.42 & 0.01 & 2.32 & -0.09 \\
\hline & $A u-C$ & 2.13 & 2.11 & -0.02 & 2.00 & -0.13 \\
\hline & C-Au-Au & 134.8 & 131.2 & -3.6 & 132.8 & -1.9 \\
\hline \multirow[t]{3}{*}{$\mathrm{AuF}_{2}(\mathrm{SH})$} & Au-S & 2.34 & 2.26 & -0.08 & 2.16 & -0.18 \\
\hline & Au-F & 2.02 & 1.99 & -0.03 & 2.04 & 0.02 \\
\hline & S-Au-F & 89.4 & 97.3 & 7.9 & 87.0 & -2.3 \\
\hline
\end{tabular}


$\mathrm{AuC}_{2} \mathrm{~N}_{4} \mathrm{H}_{8} \mathrm{~S}_{2}{ }^{+}$(AFAWUZ)

$\mathrm{AuC}_{12} \mathrm{H}_{8} \mathrm{~S}_{4}$ (JEKGAH)

$\mathrm{Au}\left(\mathrm{Me}_{2} \mathrm{~S}\right)_{2}{ }^{+}$

$\mathrm{AuCl}$

$\mathrm{AuCl}_{2}^{-}$(GANJOU)

$\mathrm{AuCl}_{3}$

$\mathrm{AuCl}_{4}^{-}$(BENYAU)

$\mathrm{AuC}_{4} \mathrm{~N}_{3} \mathrm{H}_{13} \mathrm{Cl}^{2+}$ (AEMAUP)

$\mathrm{AuC}_{2} \mathrm{H}_{6} \mathrm{SCI}$ (GOJLAS)

$\mathrm{Au}\left(\mathrm{CH}_{3}\right) \mathrm{Cl}_{2}$

$\mathrm{AuC}_{11} \mathrm{~N}_{2} \mathrm{H}_{10} \mathrm{Cl}_{2} \mathrm{O}_{2}{ }^{+}$(BATTOF)

$\mathrm{AuC}_{6} \mathrm{NH}_{3} \mathrm{Cl}_{2} \mathrm{O}_{3}$ (KUDNAY)

$\mathrm{AuC}_{5} \mathrm{NH}_{5} \mathrm{Cl}_{3}$ (PYAUCL10)

$\mathrm{AuC}_{6} \mathrm{NH}_{7} \mathrm{Cl}_{3} \mathrm{O}$ (HIHCIK)

$\mathrm{Au}_{2} \mathrm{C}_{12} \mathrm{P}_{2} \mathrm{H}_{28} \mathrm{Cl}_{2}$ (EMPLAU)

AuTi

AuV

$\mathrm{AuCr}$

AuCo

AuNi

\begin{tabular}{|c|c|c|c|}
\hline Au-S & 2.28 & 2.25 & -0.03 \\
\hline Au-S & 2.30 & 2.27 & -0.03 \\
\hline S-Au-S & 89.9 & 92.7 & 2.9 \\
\hline Au-S & 2.40 & 2.36 & -0.04 \\
\hline $\mathrm{Au}-\mathrm{Cl}$ & 2.33 & 2.26 & -0.07 \\
\hline $\mathrm{Au}-\mathrm{Cl}$ & 2.28 & 2.28 & 0.00 \\
\hline $\mathrm{Au}-\mathrm{Cl}$ & 2.32 & 2.22 & -0.10 \\
\hline $\mathrm{Au}-\mathrm{Cl}$ & 2.30 & 2.23 & -0.07 \\
\hline $\mathrm{Au}-\mathrm{Cl}$ & 2.27 & 2.25 & -0.02 \\
\hline $\mathrm{Au}-\mathrm{Cl}$ & 2.27 & 2.20 & -0.07 \\
\hline Au-N & 1.97 & 2.20 & 0.23 \\
\hline $\mathrm{N}-\mathrm{Au}-\mathrm{Cl}$ & 93.7 & 99.1 & 5.4 \\
\hline$A u-N^{\prime}$ & 2.05 & 2.26 & 0.21 \\
\hline Au-S & 2.27 & 2.35 & 0.08 \\
\hline $\mathrm{Au}-\mathrm{Cl}$ & 2.29 & 2.25 & -0.04 \\
\hline $\mathrm{Au}-\mathrm{Cl}$ & 2.35 & 2.23 & -0.12 \\
\hline $\mathrm{C}-\mathrm{Au}-\mathrm{Cl}$ & 93.0 & 93.6 & 0.6 \\
\hline $\mathrm{Cl}-\mathrm{Au}-\mathrm{Cl}$ & 177.9 & 175.7 & -2.2 \\
\hline $\mathrm{Au}-\mathrm{Cl}$ & 2.26 & 2.31 & 0.05 \\
\hline $\mathrm{Cl}-\mathrm{Au}-\mathrm{Cl}$ & 89.6 & 138.6 & 49.0 \\
\hline$A u-N$ & 2.08 & 2.36 & 0.28 \\
\hline $\mathrm{Au}-\mathrm{Cl}$ & 2.25 & 2.23 & -0.02 \\
\hline Au-N & 2.04 & 2.04 & 0.00 \\
\hline $\mathrm{Au}-\mathrm{O}$ & 1.99 & 2.07 & 0.08 \\
\hline $\mathrm{Au}-\mathrm{Cl}$ & 2.29 & 2.25 & -0.04 \\
\hline $\mathrm{Au}-\mathrm{Cl}$ & 2.26 & 2.22 & -0.04 \\
\hline $\mathrm{Au}-\mathrm{Cl}$ & 2.28 & 2.24 & -0.04 \\
\hline$A u-N$ & 2.00 & 2.25 & 0.25 \\
\hline $\mathrm{Au}-\mathrm{Cl}$ & 2.27 & 2.31 & 0.04 \\
\hline Au-N & 2.02 & 2.07 & 0.05 \\
\hline $\mathrm{Au}-\mathrm{Cl}$ & 2.38 & 2.32 & -0.06 \\
\hline$A u-C$ & 2.05 & 2.17 & 0.12 \\
\hline Cl-Au-C & 85.8 & 88.4 & 2.7 \\
\hline$A u-A u$ & 2.59 & 2.65 & 0.06 \\
\hline $\mathrm{Au}-\mathrm{Ti}$ & 2.52 & 2.52 & 0.00 \\
\hline $\mathrm{Au}-\mathrm{V}$ & 2.53 & 2.53 & 0.00 \\
\hline $\mathrm{Au}-\mathrm{Cr}$ & 2.54 & 2.54 & 0.00 \\
\hline Au-Co & 2.44 & 2.43 & 0.00 \\
\hline $\mathrm{Au}-\mathrm{Ni}$ & 2.41 & 2.40 & -0.01 \\
\hline
\end{tabular}

\begin{tabular}{|r|r|}
\hline 2.28 & 0.00 \\
\hline 2.25 & -0.05 \\
\hline 92.9 & 3.0 \\
\hline 2.30 & -0.10 \\
\hline 2.34 & 0.01 \\
\hline 2.42 & 0.14 \\
\hline 2.20 & -0.12 \\
\hline 2.06 & -0.25 \\
\hline 2.27 & 0.00 \\
\hline 2.13 & -0.14 \\
\hline 2.21 & 0.24 \\
\hline 90.5 & -3.2 \\
\hline 2.06 & 0.01 \\
\hline 2.33 & 0.06 \\
\hline 2.38 & 0.09 \\
\hline 2.31 & -0.04 \\
\hline 93.8 & 0.8 \\
\hline 177.9 & 0.0 \\
\hline 2.16 & -0.10 \\
\hline 99.3 & 9.7 \\
\hline 2.19 & 0.11 \\
\hline 2.41 & 0.16 \\
\hline 2.35 & 0.31 \\
\hline 1.42 & -0.57 \\
\hline 2.28 & -0.01 \\
\hline 2.16 & -0.10 \\
\hline 2.27 & -0.01 \\
\hline 2.17 & 0.17 \\
\hline 2.26 & -0.01 \\
\hline 2.13 & 0.11 \\
\hline 2.45 & 0.07 \\
\hline 2.23 & 0.18 \\
\hline 93.1 & 7.3 \\
3.13 & 0.54 \\
3.60 & 1.07 \\
3.60 & 1.07 \\
5.54 & 3.00 \\
3.51 & 1.08 \\
6.34 & 3.93 \\
\hline & \\
\hline
\end{tabular}


19

$\mathrm{AuCu}$
$\mathrm{AuZn}$
$\mathrm{AuZnH}$
$\mathrm{AuBr}$
$\mathrm{AuBr}_{2}{ }^{-}$(DOYMAF)
$\mathrm{AuBr}_{4}{ }^{-}$
$\mathrm{AuC}_{2} \mathrm{H}_{6} \mathrm{SBr}$ (GIGWAU)
$\mathrm{AuC}_{3} \mathrm{PH}_{9} \mathrm{Br}_{3}$ (BRMPAU)
$\mathrm{AuC}_{2} \mathrm{H}_{6} \mathrm{SBr}_{3}$ (GIGWEY)
$\mathrm{AuZrH}$
$\mathrm{AuMo}$
$\mathrm{Aul}^{-}$
$\mathrm{Aul}_{2}{ }^{-}\left(\mathrm{GANJUA}^{-}\right.$
$\mathrm{Aul}_{4}^{-}$(GEJQUH)
$\mathrm{Au}_{2} \mathrm{C}_{9} \mathrm{P}_{2} \mathrm{H}_{23} \mathrm{I}$ (BIBPIL)

$\mathrm{Au}_{2} \mathrm{C}_{6} \mathrm{P}_{2} \mathrm{H}_{18} \mathrm{I}_{2}$ (FUWFIM)

$N=113$

MSE bond length

MUE bond length

$N=22$

MSE bond angle

MUE bond angle

$\mathrm{Au}-\mathrm{Cu}$
$\mathrm{Au}-\mathrm{Zn}$
$\mathrm{Au}-\mathrm{Zn}$
$\mathrm{Au}-\mathrm{Br}$
$\mathrm{Au}-\mathrm{Br}$
$\mathrm{Au}-\mathrm{Br}$
$\mathrm{Au}-\mathrm{Br}$
$\mathrm{Au}-\mathrm{S}$
$\mathrm{Au}-\mathrm{Br}$
$\mathrm{Au}-\mathrm{Br}$
$\mathrm{Au}-\mathrm{P}$
$\mathrm{Au}-\mathrm{Br}$
$\mathrm{Au}-\mathrm{S}$
$\mathrm{Au}-\mathrm{Zr}$
$\mathrm{Au}-\mathrm{Mo}$
$\mathrm{Au}-\mathrm{I}$
$\mathrm{Au}-\mathrm{I}$
$\mathrm{Au}-\mathrm{I}$
$\mathrm{Au}-\mathrm{Au}$
$\mathrm{Au}-\mathrm{I}$
$\mathrm{Au}-\mathrm{C}$
$\mathrm{Au}-\mathrm{C}(\mathrm{P})$
$\mathrm{C}-\mathrm{Au}-\mathrm{Au}$
$\mathrm{Au}-\mathrm{I}$
$\mathrm{Au}-\mathrm{P}$

2.39
2.50
2.42
2.44
2.38
2.55
2.40
2.28
2.51
2.48
2.48
2.42
2.35
2.70
2.62
2.61
2.53
2.63
2.70
2.89
2.13
2.09
88.8
2.58
2.26

\begin{tabular}{|r|r|}
2.40 & 0.00 \\
2.58 & 0.08 \\
\hline 2.36 & -0.06 \\
\hline
\end{tabular}

$\begin{array}{ll}3.78 & 1.39\end{array}$

$2.36-0.06$

$2.30 \quad-0.15$

$2.33-0.05$

$2.35 \quad-0.20$

$2.30 \quad-0.10$

$\begin{array}{ll}2.35 & 0.07\end{array}$

$2.82 \quad 0.31$

$2.29 \quad-0.19$

$2.54 \quad 0.06$

\begin{tabular}{ll}
$2.42 \quad 0.00$ \\
\hline
\end{tabular}

$2.32-0.03$

$2.70 \quad 0.00$

$2.62 \quad 0.00$

$2.80 \quad 0.19$

$2.54 \quad 0.01$

$\begin{array}{lr}2.55 & -0.08 \\ 3.16 & 0.46\end{array}$

\begin{tabular}{r|r}
3.16 & 0.46 \\
2.53 & -0.36
\end{tabular}

$2.12-0.01$

$2.17 \quad 0.08$

$87.3 \quad-1.5$

$2.53-0.06$

$2.39 \quad 0.13$

AM1*

$2.80 \quad 0.30$

$2.80 \quad 0.38$

$2.26 \quad-0.18$

$2.43 \quad 0.05$

\begin{tabular}{ll}
2.28 & -0.27 \\
\hline
\end{tabular}

$2.34-0.06$

$\begin{array}{ll}2.32 & 0.04\end{array}$

$2.49-0.02$

$\begin{array}{ll}2.97 & 0.49\end{array}$

$2.08-0.40$

$\begin{array}{ll}2.84 & 0.42\end{array}$

$\begin{array}{ll}2.39 & 0.04\end{array}$

$\begin{array}{ll}3.19 & 0.49\end{array}$

$3.70 \quad 1.08$

$2.57-0.04$

$2.67 \quad 0.14$

$2.91 \quad 0.28$

$3.22 \quad 0.52$

$2.67-0.22$

$2.11-0.02$

$2.11 \quad 0.02$

\begin{tabular}{r|r}
121.3 & 32.5
\end{tabular}

$\begin{array}{ll}2.65 & 0.07\end{array}$

$2.32 \quad 0.06$

PM6

\begin{tabular}{l|l}
0.01 & 0.10 \\
0.07 & 0.28
\end{tabular}

3.7

6.9

$-2.1$

10.4 


\section{Figure captions}

15 Fig. 1 AM1* (upper row) and PM6 (lower row) optimized structures of neutral gold 16 clusters with 4 to 9 atoms

17

Fig.

19

AM1* (a) and PM6 (b) optimized structure of planar edge-capped triangle $\mathrm{Au}_{7}$ with $\mathrm{C}_{\mathrm{s}}$ symmetry 

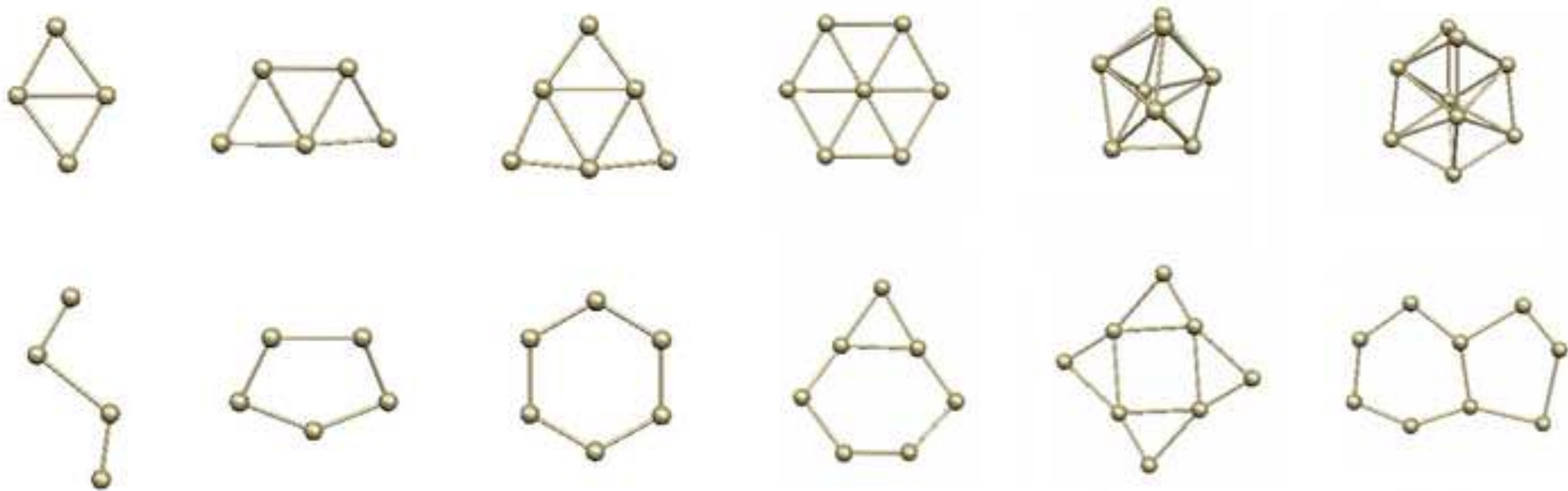
Figure 2

Click here to download high resolution image

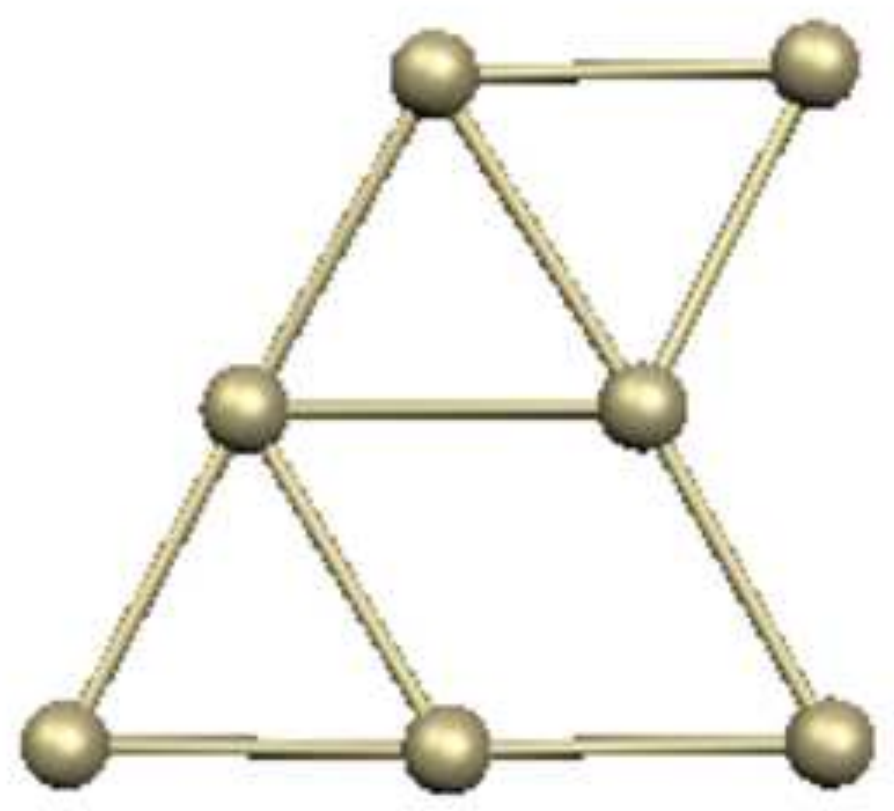

(a)

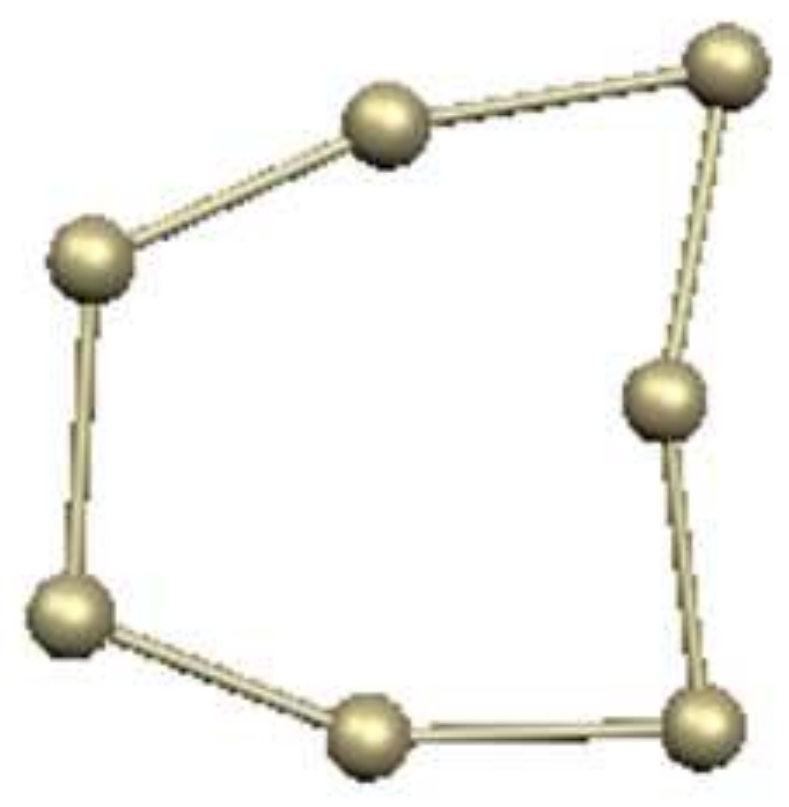

(b) 


\section{AM1* parameters for gold}

Hakan Kayi

Computer-Chemie-Centrum and Interdisciplinary Center for Molecular Materials, Friedrich-Alexander-Universität Erlangen-Nürnberg, Nägelsbachstraße 25, 91052 Erlangen, Germany

E-mail: Hakan.Kayi@chemie.uni-erlangen.de

\section{Supplementary Material}

\begin{tabular}{|l|l|c|}
\hline & & Page \\
\hline Table S1 & $\begin{array}{l}\text { Sources and calculation details for the heats of formation } \\
\text { used to parameterize. }\end{array}$ & 2 \\
\hline Table S2 & $\begin{array}{l}\text { Sources and calculation details for the ionization potentials } \\
\text { and dipole moments used to parameterize. }\end{array}$ & 3 \\
\hline Table S3 & $\begin{array}{l}\text { Sources and calculation details for the geometrical variables } \\
\text { used to parameterize. }\end{array}$ & 4 \\
\hline References & & 7 \\
\hline
\end{tabular}


Table S1: Sources and calculation details for the heats of formation used to parameterize.

\begin{tabular}{|c|c|c|c|}
\hline \multicolumn{4}{|c|}{ Heats of Formation For Gold Containing Compounds } \\
\hline Compound & $\begin{array}{c}\text { Target } \\
\left(\mathrm{kcal} \mathrm{mol}^{-1}\right)\end{array}$ & Source & $\begin{array}{c}\text { Total Energy } \\
\text { (a.u.) }\end{array}$ \\
\hline $\mathrm{Au}$ & 88.0 & 1 & \\
\hline $\mathrm{Au}^{-}$ & 20.0 & 2 & \\
\hline $\mathrm{Au}^{+}$ & 300.7 & 3 & \\
\hline $\mathrm{Au}_{2}$ & 87.0 & 3 & \\
\hline $\mathrm{AuH}$ & 78.7 & 3 & \\
\hline $\mathrm{AuH}_{3}$ & 149.1 & 4 & -137.1181929 \\
\hline $\mathrm{AuCH}_{3}$ & 67.3 & 3 & \\
\hline $\mathrm{Au}\left(\mathrm{CH}_{3}\right)_{3}$ & 84.7 & 3 & \\
\hline $\mathrm{AuNH}_{2}$ & 93.9 & 3 & \\
\hline $\mathrm{Au}\left(\mathrm{CH}_{3}\right)_{2}\left(\mathrm{NH}_{2}\right)$ & 83.9 & 3 & \\
\hline $\mathrm{Au}(\mathrm{CN})_{2}^{-}$ & 54.9 & 3 & \\
\hline $\mathrm{Au}\left(\mathrm{NH}_{2}\right)_{3}$ & 126.0 & 3 & \\
\hline $\mathrm{Au}\left(\mathrm{NH}_{3}\right)_{4}^{+}$ & 154.5 & 3 & \\
\hline $\mathrm{Au}\left(\mathrm{NH}_{3}\right)_{6}{ }^{2+}$ & 355.1 & 3 & \\
\hline$\left(\mathrm{Au}\left(\mathrm{CH}_{3}\right)_{2}(\mathrm{CN})\right)_{4}$ & 362.7 & 3 & \\
\hline $\mathrm{AuO}^{-}$ & 57.1 & 3 & \\
\hline $\mathrm{AuOH}$ & 57.8 & 3 & \\
\hline $\mathrm{Au}\left(\mathrm{CH}_{3}\right)_{2}(\mathrm{OH})$ & 45.7 & 3 & \\
\hline $\mathrm{Au}\left(\mathrm{H}_{2} \mathrm{O}\right)_{4}^{+}$ & -37.8 & 4 & -441.0047270 \\
\hline $\mathrm{Au}(\mathrm{CO})_{4}^{+}$ & 141.1 & 3 & \\
\hline AuF & 50.0 & 3 & \\
\hline $\mathrm{AuF}_{3}$ & 12.7 & 4 & -434.9204586 \\
\hline $\mathrm{AuF}_{4}^{-}$ & -79.7 & 2 & \\
\hline $\mathrm{Au}\left(\mathrm{CH}_{3}\right) \mathrm{F}_{2}$ & -10.0 & 3 & \\
\hline $\mathrm{AuAlH}_{2}$ & 90.6 & 4 & -138.6910003 \\
\hline $\mathrm{AuSiH}_{3}$ & 84.1 & 4 & -141.1827166 \\
\hline $\mathrm{AuPH}_{3}^{+}$ & 260.8 & 4 & -143.5124721 \\
\hline $\mathrm{AuSH}_{2}^{+}$ & 269.1 & 4 & -146.5020438 \\
\hline $\mathrm{AuF}_{2}(\mathrm{SH})$ & 7.6 & 3 & \\
\hline $\mathrm{Au}\left(\mathrm{Me}_{2} \mathrm{~S}\right)_{2}$ & 148.8 & 3 & \\
\hline $\mathrm{AuCl}$ & 60.2 & 3 & \\
\hline $\mathrm{AuCl}_{2}^{-}$ & -67.1 & 3 & \\
\hline $\mathrm{AuCl}_{3}$ & 65.1 & 4 & -180.3208113 \\
\hline $\mathrm{AuCl}_{4}^{-}$ & -89.7 & 3 & \\
\hline AuTi & 145.7 & 4 & -193.4954244 \\
\hline $\mathrm{AuV}$ & 100.2 & 4 & -206.7655279 \\
\hline $\mathrm{AuCr}$ & 137.0 & 4 & -221.7864528 \\
\hline AuCo & 141.5 & 4 & -280.5419878 \\
\hline $\mathrm{AuNi}$ & 77.8 & 4 & -304.7746291 \\
\hline $\mathrm{AuCu}$ & 117.7 & 4 & -331.6454290 \\
\hline $\mathrm{AuZnH}$ & 94.5 & 4 & -201.6731244 \\
\hline $\mathrm{AuBr}$ & 62.8 & 3 & \\
\hline $\mathrm{AuBr}_{2}^{-}$ & -56.3 & 3 & \\
\hline $\mathrm{AuBr}_{4}^{-}$ & -66.4 & 3 & \\
\hline $\mathrm{AuZrH}$ & 173.0 & 4 & -182.5525394 \\
\hline AuMo & 251.5 & 4 & -202.8576504 \\
\hline
\end{tabular}




\begin{tabular}{|l|r|r|l|}
\hline $\mathrm{Aul}$ & 69.0 & 3 & \\
\hline $\mathrm{Aul}_{2}^{-}$ & -38.1 & 3 & \\
\hline $\mathrm{Aul}_{4}^{-}$ & -26.8 & 3 & \\
\hline
\end{tabular}

Table S2: Sources and calculation details for the ionization potentials and dipole moments used to parameterize.

\begin{tabular}{|c|c|c|}
\hline \multicolumn{3}{|c|}{$\begin{array}{l}\text { Koopmans' Theorem lonization Potentials for Gold } \\
\text { Containing Compounds }\end{array}$} \\
\hline Compound & Target $(\mathrm{eV})$ & Source \\
\hline $\mathrm{Au}$ & 9.22 & 2 \\
\hline $\mathrm{Au}_{2}$ & 8.70 & 2 \\
\hline AuAl & 7.60 & 2 \\
\hline $\mathrm{AuAl}_{2}$ & 6.20 & 2 \\
\hline $\mathrm{Au}_{2} \mathrm{Al}$ & 7.70 & 2 \\
\hline AuSi & 9.50 & 2 \\
\hline $\mathrm{Au}\left(\mathrm{CH}_{3}\right) \mathrm{P}\left(\mathrm{CH}_{3}\right)_{3}$ & 7.70 & 2 \\
\hline $\mathrm{Au}\left(\mathrm{CH}_{3}\right)_{3} \mathrm{P}\left(\mathrm{CH}_{3}\right)_{3}$ & 7.80 & 2 \\
\hline \multicolumn{3}{|c|}{ Dipole Moments for Gold Containing Compounds } \\
\hline Compound & Target (Debye) & Source \\
\hline $\mathrm{AuH}$ & 0.95 & 3 \\
\hline $\mathrm{AuCH}_{3}$ & 0.46 & 3 \\
\hline $\mathrm{Au}\left(\mathrm{CH}_{3}\right)_{3}$ & 1.78 & 3 \\
\hline $\mathrm{AuNH}_{2}$ & 1.63 & 3 \\
\hline $\mathrm{Au}\left(\mathrm{CH}_{3}\right)_{2}\left(\mathrm{NH}_{2}\right)$ & 0.34 & 3 \\
\hline $\mathrm{Au}\left(\mathrm{NH}_{2}\right)_{3}$ & 3.02 & 3 \\
\hline $\mathrm{AuOH}$ & 2.53 & 3 \\
\hline $\mathrm{Au}\left(\mathrm{CH}_{3}\right)_{2}(\mathrm{OH})$ & 3.74 & 3 \\
\hline AuF & 4.04 & 3 \\
\hline $\mathrm{Au}\left(\mathrm{CH}_{3}\right) \mathrm{F}_{2}$ & 2.46 & 3 \\
\hline $\mathrm{AuF}_{2}(\mathrm{SH})$ & 1.60 & 3 \\
\hline $\mathrm{AuCl}$ & 3.48 & 3 \\
\hline $\mathrm{AuBr}$ & 2.87 & 3 \\
\hline Aul & 2.21 & 3 \\
\hline
\end{tabular}


Table S3: Sources and calculation details for the geometrical variables used to parameterize.

\begin{tabular}{|c|c|c|c|}
\hline \multicolumn{4}{|c|}{ Geometrical Variables for Gold Containing Compounds } \\
\hline Compound & Variable & \multirow{2}{*}{$\begin{array}{r}\text { Target } \\
2.63\end{array}$} & \multirow{2}{*}{$\begin{array}{c}\text { Source } \\
3\end{array}$} \\
\hline $\mathrm{Au}_{2}$ & $\mathrm{Au}-\mathrm{Au}$ & & \\
\hline $\mathrm{Au}_{2}^{-}$ & $\mathrm{Au}-\mathrm{Au}$ & 2.73 & 4 \\
\hline $\mathrm{AuH}$ & $\mathrm{Au}-\mathrm{H}$ & 1.52 & 3 \\
\hline \multirow{2}{*}{$\mathrm{AuH}_{3}$} & $\mathrm{Au}-\mathrm{H}$ & 1.70 & 4 \\
\hline & $\mathrm{H}-\mathrm{Au}-\mathrm{H}$ & 120.0 & 4 \\
\hline \multirow[t]{2}{*}{$\mathrm{AuCH}_{3}$} & $A u-C$ & 2.08 & 3 \\
\hline & $\mathrm{H}-\mathrm{C}-\mathrm{Au}$ & 107.2 & 4 \\
\hline \multirow[t]{3}{*}{$\mathrm{Au}\left(\mathrm{CH}_{3}\right)_{3}$} & $A u-C$ & 2.06 & 4 \\
\hline & $A u-C^{\prime}$ & 2.12 & 4 \\
\hline & C-Au-C & 176.1 & 4 \\
\hline $\mathrm{AuNH}_{2}$ & Au-N & 2.09 & 3 \\
\hline \multirow{3}{*}{$\mathrm{Au}\left(\mathrm{CH}_{3}\right)_{2}\left(\mathrm{NH}_{2}\right)$} & $A u-C$ & 2.13 & 3 \\
\hline & C-Au-C & 80.1 & 3 \\
\hline & Au-N & 2.04 & 3 \\
\hline \multirow[t]{2}{*}{$\mathrm{Au}(\mathrm{CN})_{2}^{-}$} & $A u-C$ & 2.04 & 3 \\
\hline & C-Au-C & 180.0 & 4 \\
\hline \multirow[t]{2}{*}{$\mathrm{Au}(\mathrm{CN})_{2}^{-}(\mathrm{JEYXAM})$} & Au-C & 2.02 & 5 \\
\hline & C-N & 1.11 & 5 \\
\hline \multirow{2}{*}{$\mathrm{AuC}_{3} \mathrm{~N}_{2} \mathrm{H}_{3}$ (CYMIAU) } & $A u-C(N)$ & 2.00 & 5 \\
\hline & $\mathrm{Au}-\mathrm{C}(\mathrm{NCH} 3)$ & 1.98 & 5 \\
\hline \multirow[t]{3}{*}{$\mathrm{Au}\left(\mathrm{NH}_{2}\right)_{3}$} & $A u-N$ & 2.08 & 3 \\
\hline & N-Au-N & 158.1 & 3 \\
\hline & Au-N' & 2.11 & 3 \\
\hline $\mathrm{Au}\left(\mathrm{NH}_{3}\right)_{4}{ }^{3+}$ & Au-N & 2.17 & 3 \\
\hline $\mathrm{Au}\left(\mathrm{NH}_{3}\right)_{4}^{+}$ & Au-N & 2.50 & 3 \\
\hline \multirow[t]{3}{*}{$\mathrm{AuC}_{14} \mathrm{~N}_{4} \mathrm{H}_{22}{ }^{+}$(AZEGIV) } & Au-N & 1.99 & 5 \\
\hline & Au-N' & 1.97 & 5 \\
\hline & N-Au-N & 95.6 & 5 \\
\hline \multirow[t]{2}{*}{$\mathrm{Au}\left(\mathrm{NH}_{3}\right)_{6}{ }^{2+}$} & Au-N & 2.67 & 3 \\
\hline & Au-N' & 2.24 & 3 \\
\hline \multirow[t]{3}{*}{$\mathrm{Au}_{2} \mathrm{C}_{6} \mathrm{~N}_{2} \mathrm{H}_{2} \mathrm{O}$ (DEVLUL) } & Au-N & 2.14 & 5 \\
\hline & Au-C & 2.04 & 5 \\
\hline & C-Au-N & 97.1 & 5 \\
\hline \multirow[t]{4}{*}{$\left(\mathrm{Au}\left(\mathrm{CH}_{3}\right)_{2}(\mathrm{CN})\right)_{4}$} & $A u-A u$ & 5.50 & 3 \\
\hline & $\mathrm{Au}-\mathrm{C}(\mathrm{CN})$ & 2.03 & 3 \\
\hline & Au-N & 2.14 & 3 \\
\hline & $\mathrm{Au}-\mathrm{C}(\mathrm{Me})$ & 2.11 & 3 \\
\hline $\mathrm{AuO}^{-}$ & $\mathrm{Au}-\mathrm{O}$ & 1.98 & 3 \\
\hline $\mathrm{AuOH}$ & $\mathrm{Au}-\mathrm{O}$ & 1.98 & 4 \\
\hline \multirow[t]{3}{*}{$\mathrm{Au}\left(\mathrm{CH}_{3}\right)_{2}(\mathrm{OH})$} & $\mathrm{Au}-\mathrm{O}$ & 2.09 & 3 \\
\hline & $A u-C$ & 2.10 & 3 \\
\hline & O-Au-C & 106.0 & 3 \\
\hline $\mathrm{Au}\left(\mathrm{H}_{2} \mathrm{O}\right)_{2}^{+}$ & O-Au-O & 176.8 & 3 \\
\hline \multirow{2}{*}{$\mathrm{Au}\left(\mathrm{H}_{2} \mathrm{O}\right)_{4}^{+}$} & Au-O & 2.46 & 4 \\
\hline & Au-O' & 2.45 & 4 \\
\hline $\mathrm{Au}(\mathrm{CO})_{4}{ }^{+}$ & Au-C & 2.28 & 3 \\
\hline AuF & Au-F & 2.07 & 3 \\
\hline
\end{tabular}




\begin{tabular}{|c|c|c|c|}
\hline \multirow{3}{*}{$\mathrm{Au}\left(\mathrm{CH}_{3}\right) \mathrm{F}_{2}$} & $A u-F$ & 1.96 & 4 \\
\hline & $\mathrm{Au}-\mathrm{C}$ & 2.05 & 4 \\
\hline & C-Au-F & 91.6 & 4 \\
\hline \multirow[t]{3}{*}{$\mathrm{AuF}_{3}$} & $\mathrm{Au}-\mathrm{F}$ & 2.01 & 3 \\
\hline & $\mathrm{Au}-\mathrm{F}^{\prime}$ & 2.02 & 3 \\
\hline & F-Au-F' & 95.0 & 3 \\
\hline $\mathrm{AuF}_{4}^{-}$ & $\mathrm{Au}-\mathrm{F}$ & 1.97 & 4 \\
\hline $\mathrm{AuAlH}_{2}$ & $\mathrm{Au}-\mathrm{Al}$ & 2.38 & 4 \\
\hline $\mathrm{AuSiH}_{3}$ & $\mathrm{Au}-\mathrm{Si}$ & 2.32 & 4 \\
\hline $\mathrm{AuPH}_{3}{ }^{+}$ & Au-P & 2.28 & 4 \\
\hline $\mathrm{AuPH}_{2}$ & Au-P & 2.33 & 4 \\
\hline $\mathrm{AuP}^{2-}$ & $\mathrm{Au} \equiv \mathrm{P}$ & 2.28 & 4 \\
\hline $\mathrm{Au}\left(\mathrm{PH}_{3}\right)_{2}^{+}$ & Au-P & 2.38 & 4 \\
\hline \multirow[t]{2}{*}{$\mathrm{Au}_{2} \mathrm{C}_{10} \mathrm{P}_{4} \mathrm{H}_{28}^{+}$(DUKREG01) } & Au-P & 2.30 & 5 \\
\hline & P-Au-P & 176.8 & 5 \\
\hline AuSH & $\mathrm{Au}-\mathrm{S}$ & 2.30 & 4 \\
\hline $\mathrm{AuSH}_{2}^{+}$ & $\mathrm{Au}-\mathrm{S}$ & 2.37 & 4 \\
\hline \multirow[t]{4}{*}{$\mathrm{Au}_{2} \mathrm{C}_{8} \mathrm{H}_{22} \mathrm{~S}_{2}$ (CULYIR) } & $\mathrm{Au}-\mathrm{Au}$ & 3.46 & 5 \\
\hline & $\mathrm{Au}-\mathrm{S}$ & 2.41 & 5 \\
\hline & $\mathrm{Au}-\mathrm{C}$ & 2.13 & 5 \\
\hline & C-Au-Au & 134.8 & 5 \\
\hline \multirow[t]{3}{*}{$\mathrm{AuF}_{2}(\mathrm{SH})$} & Au-S & 2.34 & 3 \\
\hline & $\mathrm{Au}-\mathrm{F}$ & 2.02 & 3 \\
\hline & S-Au-F & 89.4 & 3 \\
\hline $\mathrm{AuC}_{2} \mathrm{~N}_{4} \mathrm{H}_{8} \mathrm{~S}_{2}{ }^{+}(\mathrm{AFAWUZ})$ & Au-S & 2.28 & 5 \\
\hline \multirow[t]{2}{*}{$\mathrm{AuC}_{12} \mathrm{H}_{8} \mathrm{~S}_{4}(\mathrm{JEKGAH})$} & $\mathrm{Au}-\mathrm{S}$ & 2.30 & 5 \\
\hline & S-Au-S & 89.9 & 5 \\
\hline $\mathrm{Au}\left(\mathrm{Me}_{2} \mathrm{~S}\right)_{2}^{+}$ & $\mathrm{Au}-\mathrm{S}$ & 2.40 & 3 \\
\hline $\mathrm{AuCl}$ & $\mathrm{Au}-\mathrm{Cl}$ & 2.33 & 3 \\
\hline $\mathrm{AuCl}_{2}^{-}$(GANJOU) & $\mathrm{Au}-\mathrm{Cl}$ & 2.28 & 5 \\
\hline \multirow[t]{2}{*}{$\mathrm{AuCl}_{3}$} & $\mathrm{Au}-\mathrm{Cl}$ & 2.32 & 4 \\
\hline & $\mathrm{Au}-\mathrm{Cl}$ & 2.30 & 4 \\
\hline $\mathrm{AuCl}_{4}^{-}(\mathrm{BENYAU})$ & $\mathrm{Au}-\mathrm{Cl}$ & 2.27 & 5 \\
\hline \multirow[t]{4}{*}{$\mathrm{AuC}_{4} \mathrm{~N}_{3} \mathrm{H}_{13} \mathrm{Cl}^{2+}$ (AEMAUP) } & $\mathrm{Au}-\mathrm{Cl}$ & 2.27 & 5 \\
\hline & Au-N & 1.97 & 5 \\
\hline & $\mathrm{N}-\mathrm{Au}-\mathrm{Cl}$ & 93.7 & 5 \\
\hline & Au-N' & 2.05 & 5 \\
\hline \multirow[t]{2}{*}{$\mathrm{AuC}_{2} \mathrm{H}_{6} \mathrm{SCl}$ (GOJLAS) } & $\mathrm{Au}-\mathrm{S}$ & 2.27 & 5 \\
\hline & $\mathrm{Au}-\mathrm{Cl}$ & 2.29 & 5 \\
\hline \multirow[t]{3}{*}{$\mathrm{Au}\left(\mathrm{CH}_{3}\right) \mathrm{Cl}_{2}$} & $\mathrm{Au}-\mathrm{Cl}$ & 2.35 & 3 \\
\hline & C-Au-Cl & 93.0 & 3 \\
\hline & $\mathrm{Cl}-\mathrm{Au}-\mathrm{Cl}$ & 177.9 & 3 \\
\hline \multirow[t]{3}{*}{$\mathrm{AuC}_{11} \mathrm{~N}_{2} \mathrm{H}_{10} \mathrm{Cl}_{2} \mathrm{O}_{2}^{+}$(BATTOF) } & $\mathrm{Au}-\mathrm{Cl}$ & 2.26 & 5 \\
\hline & $\mathrm{Cl}-\mathrm{Au}-\mathrm{Cl}$ & 89.6 & 5 \\
\hline & Au-N & 2.08 & 5 \\
\hline \multirow[t]{3}{*}{$\mathrm{AuC}_{6} \mathrm{NH}_{3} \mathrm{Cl}_{2} \mathrm{O}_{3}$ (KUDNAY) } & $\mathrm{Au}-\mathrm{Cl}$ & 2.25 & 5 \\
\hline & Au-N & 2.04 & 5 \\
\hline & $\mathrm{Au}-\mathrm{O}$ & 1.99 & 5 \\
\hline \multirow[t]{4}{*}{$\mathrm{AuC}_{5} \mathrm{NH}_{5} \mathrm{Cl}_{3}(\mathrm{PYAUCL} 10)$} & $\mathrm{Au}-\mathrm{Cl}$ & 2.29 & 5 \\
\hline & $\mathrm{Au}-\mathrm{Cl}$ & 2.26 & 5 \\
\hline & $\mathrm{Au}-\mathrm{Cl}$ & 2.28 & 5 \\
\hline & Au-N & 2.00 & 5 \\
\hline $\mathrm{AuC}_{6} \mathrm{NH}_{7} \mathrm{Cl}_{3} \mathrm{O}(\mathrm{HIHCIK})$ & $\mathrm{Au}-\mathrm{Cl}$ & 2.27 & 5 \\
\hline
\end{tabular}




\begin{tabular}{|c|c|c|c|}
\hline & Au-N & 2.02 & 5 \\
\hline \multirow[t]{4}{*}{$\mathrm{Au}_{2} \mathrm{C}_{12} \mathrm{P}_{2} \mathrm{H}_{28} \mathrm{Cl}_{2}$ (EMPLAU) } & $\mathrm{Au}-\mathrm{Cl}$ & 2.38 & 5 \\
\hline & Au-C & 2.05 & 5 \\
\hline & $\mathrm{Cl}-\mathrm{Au}-\mathrm{C}$ & 85.8 & 5 \\
\hline & $\mathrm{Au}-\mathrm{Au}$ & 2.59 & 5 \\
\hline AuTi & Au-Ti & 2.52 & 4 \\
\hline $\mathrm{AuV}$ & $A u-V$ & 2.53 & 4 \\
\hline $\mathrm{AuCr}$ & $\mathrm{Au}-\mathrm{Cr}$ & 2.54 & 4 \\
\hline AuCo & $\mathrm{Au}-\mathrm{Co}$ & 2.44 & 4 \\
\hline $\mathrm{AuNi}$ & $\mathrm{Au}-\mathrm{Ni}$ & 2.41 & 4 \\
\hline $\mathrm{AuCu}$ & $\mathrm{Au}-\mathrm{Cu}$ & 2.39 & 4 \\
\hline AuZn & $A u-Z n$ & 2.50 & 4 \\
\hline $\mathrm{AuZnH}$ & $A u-Z n$ & 2.42 & 4 \\
\hline $\mathrm{AuBr}$ & $\mathrm{Au}-\mathrm{Br}$ & 2.44 & 3 \\
\hline $\mathrm{AuBr}_{2}^{-}$(DOYMAF) & $\mathrm{Au}-\mathrm{Br}$ & 2.38 & 5 \\
\hline $\mathrm{AuBr}_{4}^{-}$ & $\mathrm{Au}-\mathrm{Br}$ & 2.55 & 4 \\
\hline \multirow[t]{2}{*}{$\mathrm{AuC}_{2} \mathrm{H}_{6} \mathrm{SBr}$ (GIGWAU) } & $\mathrm{Au}-\mathrm{Br}$ & 2.40 & 5 \\
\hline & Au-S & 2.28 & 5 \\
\hline \multirow[t]{3}{*}{$\mathrm{AuC}_{3} \mathrm{PH}_{9} \mathrm{Br}_{3}$ (BRMPAU) } & $\mathrm{Au}-\mathrm{Br}$ & 2.51 & 5 \\
\hline & $\mathrm{Au}-\mathrm{Br}^{\prime}$ & 2.48 & 5 \\
\hline & Au-P & 2.48 & 5 \\
\hline \multirow[t]{2}{*}{$\mathrm{AuC}_{2} \mathrm{H}_{6} \mathrm{SBr}_{3}$ (GIGWEY) } & $\mathrm{Au}-\mathrm{Br}$ & 2.42 & 5 \\
\hline & Au-S & 2.35 & 5 \\
\hline $\mathrm{AuZrH}$ & $\mathrm{Au}-\mathrm{Zr}$ & 2.70 & 4 \\
\hline AuMo & Au-Mo & 2.62 & 4 \\
\hline Aul & $A u-I$ & 2.61 & 3 \\
\hline $\mathrm{Aul}_{2}^{-}$(GANJUA) & $\mathrm{Au}-\mathrm{I}$ & 2.53 & 5 \\
\hline $\mathrm{Aul}_{4}^{-}$(GEJQUH) & $A u-I$ & 2.63 & 5 \\
\hline \multirow[t]{5}{*}{$\mathrm{Au}_{2} \mathrm{C}_{9} \mathrm{P}_{2} \mathrm{H}_{23} \mathrm{I}$ (BIBPIL) } & $\mathrm{Au}-\mathrm{Au}$ & 2.70 & 5 \\
\hline & Au-I & 2.89 & 5 \\
\hline & Au-C & 2.13 & 5 \\
\hline & $A u-C(P)$ & 2.09 & 5 \\
\hline & C-Au-Au & 88.8 & 5 \\
\hline \multirow[t]{2}{*}{$\mathrm{Au}_{2} \mathrm{C}_{6} \mathrm{P}_{2} \mathrm{H}_{18} \mathrm{l}_{2}$ (FUWFIM) } & $A u-I$ & 2.58 & 5 \\
\hline & Au-P & 2.26 & 5 \\
\hline
\end{tabular}




\section{REFERENCES}

[S1] Lide D.R. (Ed.) (2006) CRC Handbook of Chemistry and Physics, 87th edn. Taylor and Francis CRC Press, Boca Raton, FL

[S2] NIST Chemistry WebBook, NIST Standard Reference Database Number 69, (http://webbook.nist.gov/), Linstrom P., Mallard W. National Institute of Standards and Technology: Gaithersburg MD, 20899, 2003

[S3] Stewart JJP (2007) J. Mol. Model. 13:1173-1213

[S4] B3LYP/LANL2DZ with polarization functions, see main text for references.

[S5] Cambridge Structural Database, Version 5.28 (2007) Cambridge Crystallographic Data Centre, Cambridge, UK 